\title{
Coupled microvibration analysis of a reaction wheel assembly including gyroscopic effects in its accelerance
}

\author{
Zhe Zhang ${ }^{\mathrm{a}, *}$, Guglielmo S. Aglietti ${ }^{\mathrm{b}}$, Weijia Ren ${ }^{\mathrm{c}}$ \\ astronautics Research Group, University of Southampton, Southampton SO17 1BJ, United Kingdom \\ ${ }^{\mathrm{b}}$ Surrey Space Centre, University of Surrey, Guildford GU2 7XH, United Kingdom \\ ' Specialized Technologies Group, Technology and Engineering Center for Space Utilization, Chinese Academy of Sciences, Beijing 100094, \\ People's Republic of China
}

\section{A R T I C L E I N F O}

\section{Article history:}

Received 12 September 2012

Received in revised form

23 May 2013

Accepted 10 June 2013

Handling Editor: H. Ouyang

\begin{abstract}
A B S T R A C T
This article discusses the coupled microvibration analysis of a cantilever configured Reaction Wheel Assembly with soft-suspension system. A RWA-seismic mass coupled microvibration measurement system is presented and its model validated against test results. The importance of the RWA driving point accelerances in coupled microvibration analysis is thoroughly discussed. A RWA accelerance measurement system has been designed to measure the driving point accelerances in both static (flywheel not spinning) and dynamic (flywheel spinning) conditions. Analytically, RWA static accelerance is obtained by frequency response analysis of a finite element model. The traditionally ignored gyroscopic effects in the accelerances are included in the model and their effects with respect to traditional models are shown both theoretically and experimentally. Although at high angular speed, when nonlinearities in the microvibrations prevent an accurate simulation, it is shown that the predicted microvibrations match more closely with the test results when considering gyroscopic effects in RWA accelerances than those predicted using the traditional method. The presented coupled microvibration analysis method is also very efficient in practice and is applicable in an industrial environment.

(c) 2013 Elsevier Ltd. All rights reserved.
\end{abstract}

\section{Introduction}

In recent years satellite microvibrations and related issues have become increasingly important in the design of scientific satellites (e.g. SOLAR-B, GOCE, SDO, JWST, SIM, etc.) as they often carry highly vibration-sensitive instruments, resulting in stringent requirements for the satellite platform stability. Microvibrations are termed as low level mechanical vibrations usually in the range of micro-g's ( $\mu \mathrm{g}$ ) and typically occur at frequencies from a few $\mathrm{Hz}$ up to $1 \mathrm{kHz}[1]$. They are usually generated by internal mechanisms on board satellites, such as Reaction Wheel Assemblies (RWAs), Momentum Wheel Assemblies (MWAs), cryocoolers, pointing mechanisms, thrusters, etc., which in this context are called microvibration sources [2]. The microvibrations caused by such sources are transmitted through the satellite structure, exciting modes of the structure or elements of the instrument and affecting its performance [3]. The items of interest excited by the microvibrations are termed receivers. The dynamics of the microvibration sources will also couple with those of the satellite structure making more complicated the prediction of microvibration effects [4].

\footnotetext{
* Corresponding author. Tel.: +44 23 80594896; fax: +44 2380593058

E-mail addresses: zhezhang8311@gmail.com (Z. Zhang), g.aglietti@surrey.ac.uk (G. S. Aglietti), renweijia@csu.ac.cn (W. Ren).
} 


\begin{tabular}{|c|c|c|c|}
\hline \multicolumn{2}{|c|}{ Nomenclature } & M & the generalized mass matrix \\
\hline \multicolumn{2}{|c|}{ Latin variables } & & $\begin{array}{l}\text { WA-seismic mass interface moment about } \\
x_{c} \text {-axis from microvibration tests }\end{array}$ \\
\hline$a_{i}$ & $\begin{array}{l}\text { accelerations from the } i \text { th accelerometers, } \\
i=1-8\end{array}$ & \multicolumn{2}{|c|}{$\begin{array}{l}M_{x}, M_{y}, M_{z} \text { moment about the generalized } x, y, z \text {-axis } \\
\text { respectively }\end{array}$} \\
\hline $\mathbf{A}_{\mathrm{c}}$ & $\begin{array}{l}l=1-8 \\
\text { seismic mass driving point accelerances }\end{array}$ & $n$ & number of harmonic \\
\hline $\boldsymbol{A}_{s}$ & matrix & & $\begin{array}{l}\text { the generalized displacement vector } \\
\text { accelerometer radius }\end{array}$ \\
\hline $\mathbf{A}_{w}$ & WA driving point accelerances matrix & $t$ & time \\
\hline$A_{w}$ & $\begin{array}{l}\text { the element in the WA accelerance } \\
\text { matrix }\end{array}$ & & $\begin{array}{l}\text { potential energy } \\
\text { generalized displacements }\end{array}$ \\
\hline$c_{t}, c_{r}, c_{z}$ & $\begin{array}{l}\text { WA suspension system damping in in-plane } \\
\text { translational, rotational and axial translational }\end{array}$ & & \\
\hline & $\begin{array}{l}\text { translational, rotational and axial translational } \\
\text { dof respectively }\end{array}$ & Greek va & riables \\
\hline C & the generalized damping matrix & & \\
\hline & $\begin{array}{l}\text { amplitude coefficient of the } i \text { th harmonic } \\
\text { the distance from flywheel CoM to the } \\
\text { soft-suspension system }\end{array}$ & $\begin{array}{l}\theta, \varphi, \psi \\
\boldsymbol{\Phi}_{F F_{-} \text {coupl }}\end{array}$ & $\begin{array}{l}\text { ed spectral density matrix of WA coupled } \\
\text { microvibrations }\end{array}$ \\
\hline$d_{i}$ & $\begin{array}{l}\text { vertical or horizontal distance between the } i \text { th } \\
\text { accelerometer to the seismic mass CoM, } \\
i=1-4\end{array}$ & $\begin{array}{l}\boldsymbol{\Phi}_{F F_{-} \text {groun }} \\
\boldsymbol{\Phi}_{\text {ZZ_receiv }}\end{array}$ & $\begin{array}{l}\text { ded spectral density matrix of WA } \\
\text { hard-mounted microvibrations } \\
\text { en spectral density matrix of receiver }\end{array}$ \\
\hline$d_{i j}$ & $\begin{array}{l}\text { distances between accelerometers } i \text { and } j \text { in } \\
\text { WA accelerance tests }\end{array}$ & & $\begin{array}{l}\text { performance } \\
\text { element in the spectral density matrix }\end{array}$ \\
\hline $\mathbf{F}$ & the generalized forcing vector & & flywheel spin speed \\
\hline$F_{x}, F_{y}, F_{z}$ & $\begin{array}{l}\text { forces in the generalized } x, y, z \text {-axis } \\
\text { respectively }\end{array}$ & & $\begin{array}{l}\text { frequency } \\
\text { damping ratio }\end{array}$ \\
\hline$F_{y i}, F_{z i}$ & $\begin{array}{l}\text { WA-seismic mass interface forces, in } y_{c} \text { and } \\
z_{c} \text {-axis respectively from microvibration tests } \\
\text { the generalized gyroscopic matrix }\end{array}$ & Sub/supe & r-scripts \\
\hline $\mathbf{G}_{f}$ & $\begin{array}{l}\text { "load filer" matrix between WA hard-mounted } \\
\text { and coupled microvibrations }\end{array}$ & $\begin{array}{l}\text { at } \\
c\end{array}$ & $\begin{array}{l}\text { axial translational dof } \\
\text { seismic mass }\end{array}$ \\
\hline $\mathbf{G}_{Z F}$ & $\begin{array}{l}\text { transfer matrix on satellite structure from WA } \\
\text { to receiver }\end{array}$ & & $\begin{array}{l}\text { in-plane rotational dof } \\
\text { in-plane translational dof }\end{array}$ \\
\hline$G_{f}$ & the element in "load filter" matrix & $s$ & WA-seismic mass system \\
\hline$h$ & $\begin{array}{l}\text { distance from WA suspension system to WA } \\
\text { base CoM }\end{array}$ & & $\begin{array}{l}\text { flywheel-base system } \\
\text { wheel assembly }\end{array}$ \\
\hline $\begin{array}{l}h_{i} \\
\text { I }\end{array}$ & $\begin{array}{l}\text { harmonic number of the } i \text { th harmonic } \\
\text { unit matrix }\end{array}$ & $R$ & total in WA "rocking" dof \\
\hline$I_{C_{-} x x}$ & $\begin{array}{l}\text { moment of inertia about } x_{c} \text {-axis of the seismic } \\
\text { mass }\end{array}$ & Abbrevia & tions \\
\hline$I_{r}$ & flywheel transverse moment of inertia & CoM & center of mass \\
\hline$I_{z}$ & flywheel polar moment of inertia & CSD & cross-spectral density \\
\hline & imaginary unit & dof & degree of freedom \\
\hline & the generalized stiffness matrix & EoM & equation of motion \\
\hline$k_{t}, k_{r}, k_{z}$ & $\begin{array}{l}\text { WA suspension system stiffness in in-plane } \\
\text { translational, rotational and axial translational } \\
\text { dof respectively }\end{array}$ & $\begin{array}{l}\text { FE } \\
\text { H1 } \\
\text { MWA }\end{array}$ & $\begin{array}{l}\text { finite element } \\
\text { fundamental harmonic } \\
\text { momentum wheel assembly }\end{array}$ \\
\hline$l$ & $\begin{array}{l}\text { vertical distance from WA soft-suspension } \\
\text { system to seismic mass CoM }\end{array}$ & $\begin{array}{l}\text { PSD } \\
\text { RWA }\end{array}$ & $\begin{array}{l}\text { power spectral density } \\
\text { reaction wheel assembly }\end{array}$ \\
\hline & mass of the seismic mass & SMVMS & seismic mass vibration measurement system \\
\hline$m_{w}$ & flywheel mass & WA & wheel assembly \\
\hline
\end{tabular}

The first step towards satellite microvibration analysis is to characterize the potential microvibration sources. Of the various microvibration sources on satellites, RWAs and MWAs (here referred together as Wheel Assemblies or WAs) are often considered among the most important [5]. WA-induced microvibrations are usually characterized from two different approaches with respect to the boundary condition: hard-mounted (or isolated, grounded, blocked) and coupled (i.e. mounted on some structure whose dynamics are affecting the measurements). Modeling and measurement methods for characterizing WA hard-mounted microvibrations have been developed extensively in the literature, for example [6-15], but research of WA-structure coupled microvibrations are not as mature. For this reason, the analysis of microvibrations 
utilizing hard-mounted microvibration emissions as direct inputs for the satellite system level microvibration analysis is called the "standard microvibration analysis method". This analysis is generally quick and is typically able to provide satellite performance predictions with acceptable accuracy, and is currently used for most microvibration analysis. However, its fundamental concept is flawed as hard-mounted microvibrations (obtained either experimentally, analytically, or a combination of the two) are not correct representations when the WA is mounted on the satellite structure. When a WA excites its mounting structure, the structure itself also excites the WA in turn, and so forth, creating a coupled motion between the two bodies thus that the loads at the boundary are different from those produced by the same WA when hardmounted. Alternatively, the WA-structure interface acceleration is non-zero and depends on the WA's properties as well as those of the satellite structure. This acceleration produces further loads that must be summed to those obtained with the WA hard-mounted.

Although the basic theory is well known, the issues related to the WA-structure coupled microvibrations were first reported in [16], where both the WA and structure were considered as rigid bodies with internal flexibilities. Concepts of the coupled microvibration modeling were laid down and relationships between forces/moments generated by the WA and performances of the structure were then derived. It was also realized that the frequency-dependent qualities (dynamic mass or its inverse, accelerance) of the WA and structure were critical in coupled microvibration analysis. The work was further expanded in [17,18], where an experimental method was developed to measure the driving point dynamic mass of a WA in a static condition (here static refers to a WA with a non-spinning flywheel). The coupled microvibrations were predicted, with results clearly showing the advantages of the coupled microvibration analysis method over the standard one. Similar works were also seen in [19,20]. However, even the coupled microvibration method developed in these works is flawed, as it only considered a WA dynamic mass with a nonspinning flywheel, which was then used to predict the coupled microvibrations at various spin speeds. When the flywheel is spinning however, not only does this produce microvibrations (e.g. because of its mass imbalances, etc.) but also the dynamic mass of the WA varies as functions of spin speed. Owing to gyroscopic effects, the WA dynamic mass becomes quite dissimilar from that of the non-spinning WA as spin speed changes. The method that uses the accelerance for a non-spinning WA to evaluate the coupled structure responses is referred here as the "traditional coupled microvibration analysis method". In [21-23], an analytical method was developed to obtain the WA accelerances with gyroscopic effects, i.e. the WA dynamic accelerances. Analytical expressions of dynamic accelerances of a WA were derived based on the WA hard-mounted microvibration model and considered only the flywheel properties (i.e. mass and moment of inertias). Although gyroscopic effects were included in the analytical expressions using this method, the internal modes of vibration of the WA were not represented, thus limiting the quality of predictions. Even though, the coupled microvibrations predicted were significantly improved from those predicted with WA static accelerances. There are no other methods for WA-structure coupled microvibration analysis.

In this article, a rigid seismic mass is used as the supporting structure for a cantilever configured WA. WA-seismic mass coupled microvibrations are measured experimentally by a bespoke measurement system. A previously derived WA-seismic mass coupled microvibration model is also fully validated with the measured microvibrations. The coupled WA-seismic mass model was derived using an energy method based on the WA hard-mounted microvibration model that was presented in $[14,15]$. Characteristics of the WA-seismic mass coupled microvibrations are discussed in this article and both experimental and analytical results are used for accelerance studies. All these works are presented in Section 2. In Section 3 studies of the WA driving point accelerances are fully discussed. The theory of WA-structure coupled microvibrations is briefly introduced. The WA driving point static accelerances are obtained analytically from the WA Finite Element (FE) model and also experimentally using a specially designed WA accelerance measurement system. The measurement system utilizes common force sensors and accelerometers as transducers; WA driving point accelerances in all six degrees of freedom (dofs) can be retrieved from the measured data. Analytical expressions of the WA driving point dynamic accelerances are derived from the WA disturbance model in a "free-free" boundary condition and considering WA internal modes. The coupled microvibrations are predicted with both WA static and dynamic accelerances, and results are compared between each other and also to the coupled test results. Discussions of the coupled microvibration analysis method developed in this article are provided. Finally the conclusion of the work is given in Section 4.

\section{WA-seismic mass coupled microvibrations}

\subsection{Background}

The quantification of WA-induced microvibrations is one of the most significant steps in assessing the suitability of a satellite's structural design when considering microvibrations. Here we focus on the coupled microvibrations induced by a cantilever configured WA. The WA is designed with a soft-suspension system; an alternative to the traditional rigid support (see Fig. 1). The WA can be used as a RWA or MWA with a maximum design spin speed up to about 10,000 rev/min.

In the WA-seismic mass coupled system, the WA is mounted on a rigid seismic mass. Experimentally, a measurement system is designed to measure WA-induced microvibrations (see Fig. 2(a)). The measurement system developed here is an improved version of the "Seismic Mass Vibration Measurement System (SMVMS)" presented in [14].

The SMVMS coordinate system is defined as $x_{s} y_{s} z_{s}$. The seismic mass coordinate system is defined as $x_{c} y_{c} z_{c}$ at the seismic mass center of mass (CoM), C. $\theta_{c}, \varphi_{c}$ and $\psi_{c}$ are the corresponding rotations about the three orthogonal axes. The WA body frame, $x_{w} y_{w} z_{w}$, is defined at the flywheel CoM, $O$. Rotations about the three axes are $\theta_{w}, \varphi_{w}$ and $\psi_{w}$. If we assume that the flywheel rotates at a constant angular speed, then $\Omega=\dot{\psi}_{w}, \Omega$ is the spin speed. $l$ is the vertical distance from the WA 

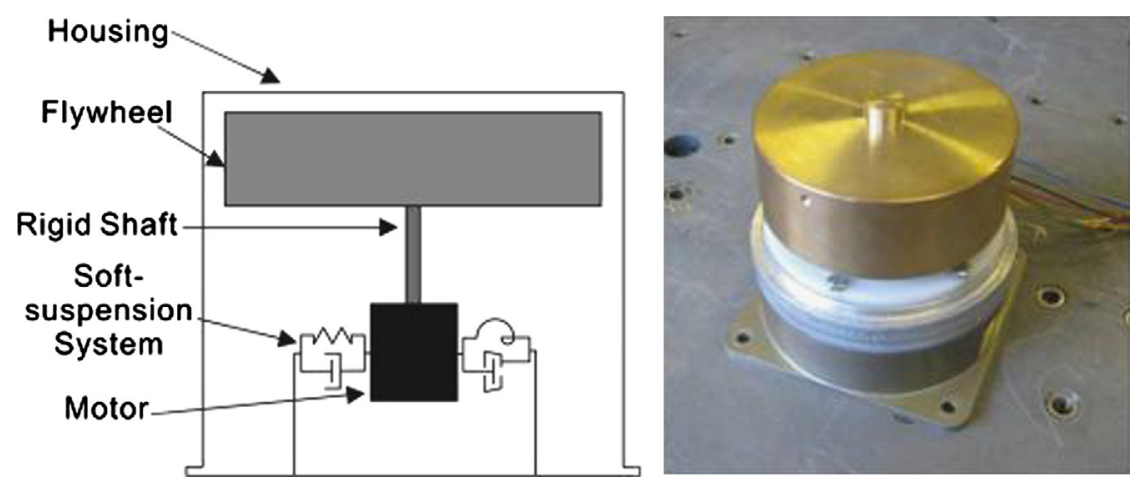

Fig. 1. Cantilever configured WA with soft-suspension system.

(a)

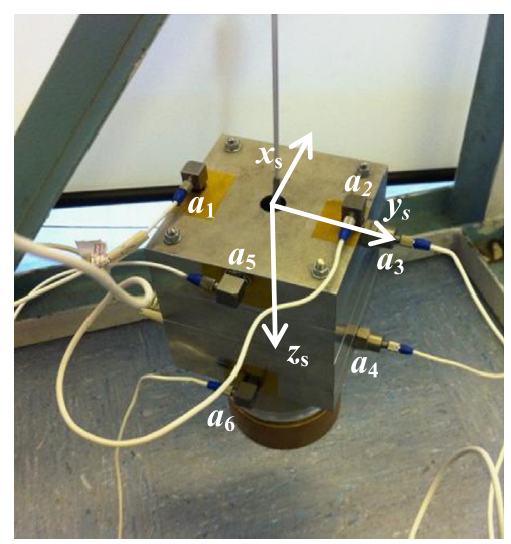

(b)

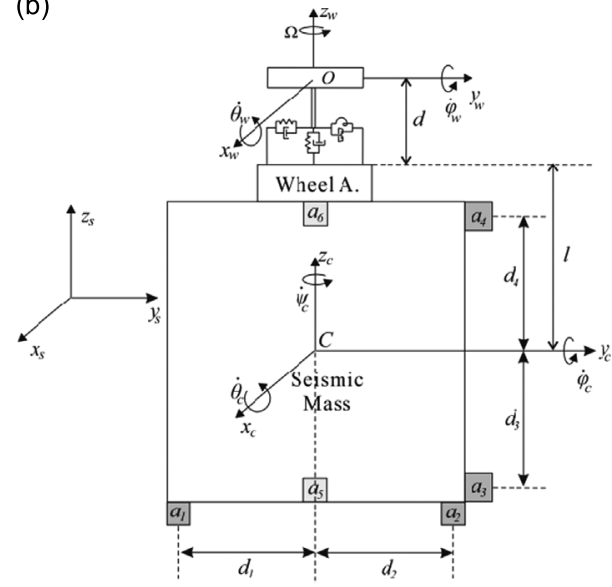

Fig. 2. The SMVMS and its simplified model: (a) test setup and (b) simplified model (shown upside down).

soft-suspension system to $C$; $d$ is the distance from $O$ to the soft-suspension system. $a_{1}$ to $a_{6}$ are the measured accelerations from accelerometers mounted on the seismic mass. $d_{1}$ to $d_{4}$ are the distances between each pair of accelerometers (see Fig. 2 (b) for detail). The SMVMS is suspended using elastic cords to simulate the "free-free" boundary condition. Accelerometers used are from PCB Model 333B50 (sensitivity $1000 \mathrm{mv} / \mathrm{g}$ and linear amplitude responses deviation \pm 5 percent up to $3000 \mathrm{~Hz}$ ) for $a_{1}, a_{2}, a_{5}$ and $a_{6}$, Endevco $752 \mathrm{~A} 13$ (sensitivity around $1000 \mathrm{mv} / \mathrm{g}$ and linear amplitude responses deviation \pm 5 percent up to $4000 \mathrm{~Hz}$ ) for $a_{3}$ and $a_{4}$.

A coupled microvibration measurement method has been developed in previous work [14]. In summary, this system allows calculation of the forces and moments at the interface between WA and seismic mass from response accelerations on the seismic mass. Their relationships in in-plane $\left(y_{s} z_{s}\right.$-plane) dofs are shown as following:

$$
\left[\begin{array}{c}
F_{y i} \\
M_{x i}
\end{array}\right]=\left[\begin{array}{cc}
\frac{m_{c} d_{4}}{d_{3}+d_{4}} & \frac{m_{c} d_{3}}{d_{3}+d_{4}} \\
\frac{I_{c} x x}{m_{c}+m_{4}\left(d_{4}+r_{a}\right)} & -\frac{I_{c, x x}-m_{c} d_{3}\left(d_{4}+r_{a}\right)}{d_{3}+d_{4}}
\end{array}\right]\left[\begin{array}{l}
a_{3} \\
a_{3}+d_{4}
\end{array}\right]
$$

where $F_{y i}$ and $M_{x i}$ are the interface forces and moments in in-plane, $a_{3}$ and $a_{4}$ are the measured accelerations. In Eq. (1), $m_{c}$ is the mass of the seismic mass, $I_{c_{-} x x}$ is the moment inertia of the seismic mass about $x_{c}$-axis, $r_{a}$ is the accelerometer radius.

The axial translational force, $F_{z i}$, at the interface can be simply expressed as

$$
F_{z i}=-\frac{m_{c}}{2}\left(a_{1}+a_{2}\right)
$$

where $a_{1}$ and $a_{2}$ are the measured accelerations.

The equations of motion (EoMs) of the WA-seismic mass coupled system have also been derived in previous work [14]. Here torque microvibrations are diminutive and are therefore ignored, thus the EoMs have ten dofs: five describing the motion of the WA, and the other five that of the seismic mass. The EoMs of this coupled system in matrix form is expressed as following:

$$
\mathbf{M}_{s} \ddot{\mathbf{q}}_{s}+\left(\mathbf{C}_{s}+\mathbf{G}_{s}\right) \dot{\mathbf{q}}_{s}+\mathbf{K}_{s} \mathbf{q}_{s}=\mathbf{F}_{s}
$$


where $\mathbf{M}_{s}, \mathbf{C}_{s}, \mathbf{K}_{s}$ and $\mathbf{G}_{s}$ are the mass matrix, damping matrix, stiffness matrix and gyroscopic matrix of the system, all have the dimension $10 \times 10$. $\mathbf{q}_{s}$ is the $10 \times 1$ vector of generalized coordinates, $\mathbf{F}_{s}$ is the $10 \times 1$ forcing vector. Elements of the matrices and vectors are given in Appendix A.

Eq. (3) is solved numerically using the state space approach with parameters taken from [14]. The detailed model derivations are not presented in this article, while test results and further model validations are discussed in Section 2.3.

\subsection{Coupled microvibration test}

WA-seismic mass coupled microvibration tests were performed using the system shown in Fig. 2(a). The WA was spun from 240 to $4980 \mathrm{rev} / \mathrm{min}$ with a step of $60 \mathrm{rev} / \mathrm{min}$. The sampling frequency was set at $2048 \mathrm{~Hz}$. The measured accelerations were transformed using Eqs. (1) and (2) to obtain the interface forces and moments. Test results of $F_{y i}, M_{x i}$ and $F_{z i}$ are plotted as spectral maps in Fig. 3 respectively.

The WA structural modes in the coupled boundary condition are first identified from the figure. In Fig. 3(a), the WA in-plane translational mode is identified at approximately $65 \mathrm{~Hz}$, while the "V" shape "rocking" mode is clearly presented in Fig. 3(b) and begins at about $27 \mathrm{~Hz}$. The axial translational mode is identified at about $46 \mathrm{~Hz}$ in Fig. 3(c). The mode visible as a vertical line in Fig. 3(a) and (b) around $220 \mathrm{~Hz}$ is due to the motor internal components and is extraneous to this discussion. It is also noticed from Fig. 3 that strong nonlinearities in responses appeared at speeds above $3060 \mathrm{rev} / \mathrm{min}$ in in-plane dofs (see Fig. 3(a) and (b) for the horizontal belts), but they are not significant in axial translational dof.

From the test results, nonlinearity has several influences on the coupled microvibrations. First of all, the primary resonances in in-plane dofs in Fig. 3(a) and (b) occur at approximately $51 \mathrm{~Hz}$ rather than at the location where the in-plane translational mode interacts with fundamental harmonics (about $65 \mathrm{~Hz}$ ), i.e. they are shifted. Secondly, responses grow at much higher rates after the resonances and fluctuate due to the "after effects" of nonlinearity. Since accelerometers operate in the linear range, nonlinearity observed in the responses is mainly due to the WA internal motor-bearing system, but as it is not the main concern in this article and its mechanics are not discussed further.

Fundamental harmonic (or $\mathrm{H} 1$ ) responses of $F_{y i}, M_{x i}$ and $F_{z i}$ are extracted from the microvibration test results, they are shown in Fig. 5 respectively in Section 2.3. In Fig. 5(a) and (b) the H1 responses start growing smoothly (roughly proportional to spin speed squared) until resonances, after that they decrease at a much slower rate and begin to fluctuate at

(a)

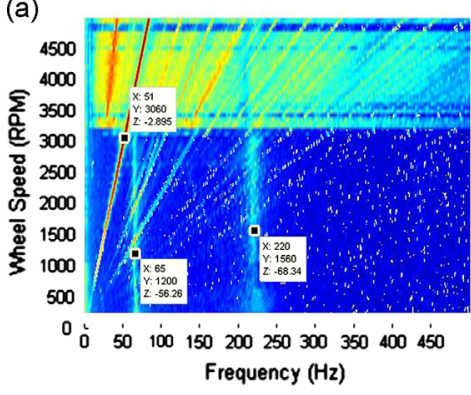

(b)

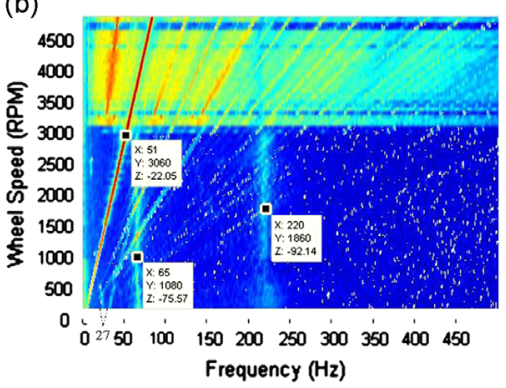

(c)

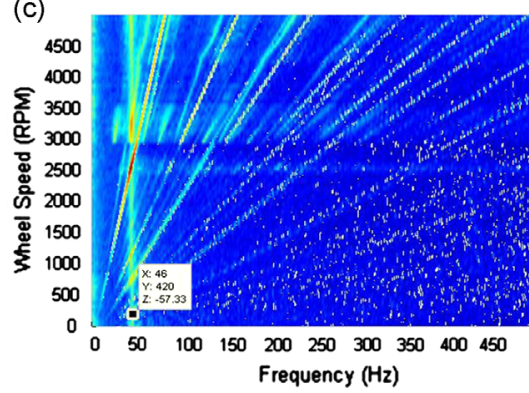

Fig. 3. Spectral maps of WA-seismic mass coupled microvibration test results: (a) $F_{y i}$; (b) $M_{x i}$; and (c) $F_{z i}$.

(a)

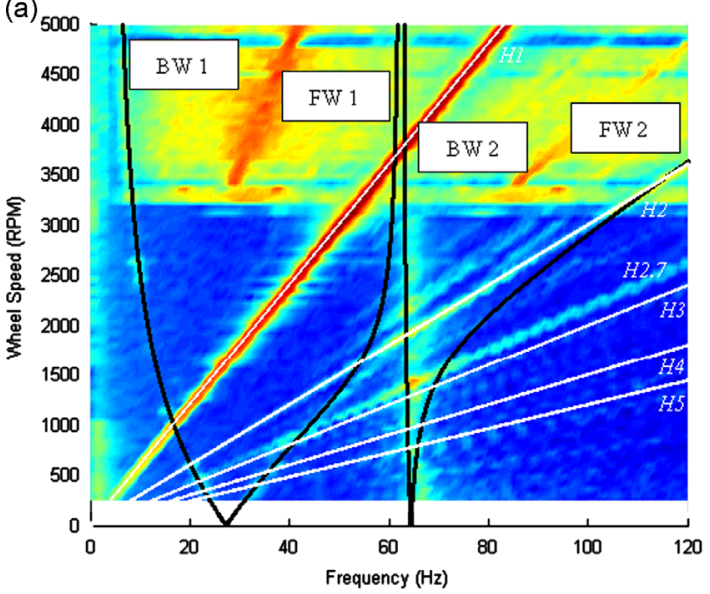

(b)

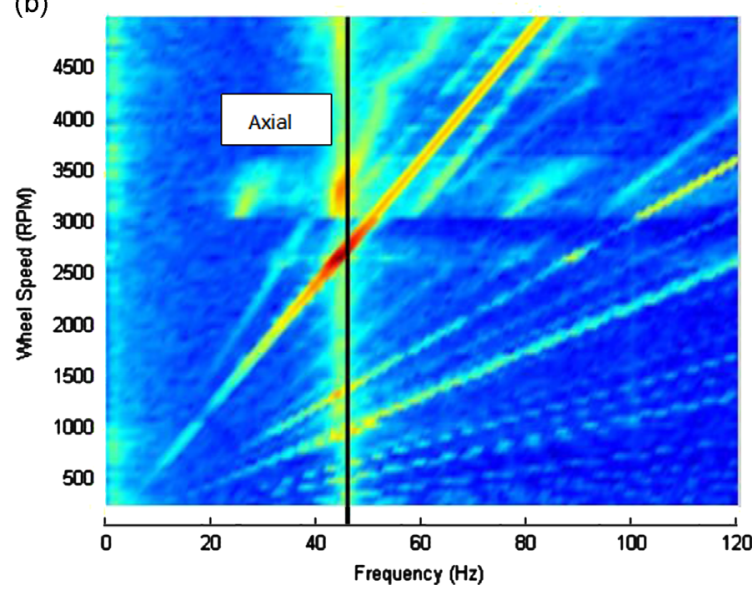

Fig. 4. WA structural modes in coupled boundary condition: (a) in-plane dofs and (b) axial translational dof. 
high spin speeds due to nonlinearity. On the other hand, resonant amplitudes in in-plane dofs are severely limited by significant damping from the WA soft-suspension system, which is one of the design features of this WA. Comparing $F_{z i}$ in Fig. 5(c), a sharp spike appears in the test results due to the reduced damping of the soft-suspension system in the axial translational dof. Note in Fig. 3, the H0.5 and H2.7, etc. are insignificant in response amplitudes compared to those of fundamental harmonics. Their apparent appearances in the figure are due to the logarithmic scale used.

\subsection{Coupled microvibration model validation}

WA structural modes in coupled boundary condition are calculated from Eq. (3) under free and undamped assumptions. They are superimposed with WA-seismic mass coupled microvibration test results $M_{x i}$ and $F_{z i}$ to validate modes in in-plane and axial translational dofs respectively (see Fig. 4(a) and (b)).

Generally speaking, all calculated structural modes have closely matched the test results. Due to the coupled motions between in-plane translational and rotational dofs for the cantilever configured WA, four speed-dependent whirls appeared in Fig. 4(a): the first backward whirl (BW1), the first forwarded whirl (FW1), the second backward whirl (BW2) and the second forward whirl (FW2). As BW2 is almost a vertical line in the figure, it is considered as the WA in-plane translational mode for parameter extraction purposes. The axial translational mode in Fig. 4(b) is constant at all times and decoupled from in-plane modes.

$\mathrm{H} 1$ responses of $F_{y i}, M_{x i}$ and $F_{z i}$ are simulated from Eq. (3) with the validated parameters such as damping ratios taken from previous work [19]. Damping ratios are $0.2,0.15$ and 0.02 respectively for $F_{y i}, M_{x i}$ and $F_{z i}$, which are extracted from each independent mode from the test results (i.e. in-plane translational mode, in-plane "rocking" mode and axial translational mode). The simulated $\mathrm{H} 1$ responses are compared to the corresponding test results in Fig. 5.

The simulated $\mathrm{H} 1$ responses in Fig. 5 match well with the test results in all cases, particularly before the resonances. As mentioned previously, at resonances, there are slight differences in the resonant frequencies between the simulated and test

(a)

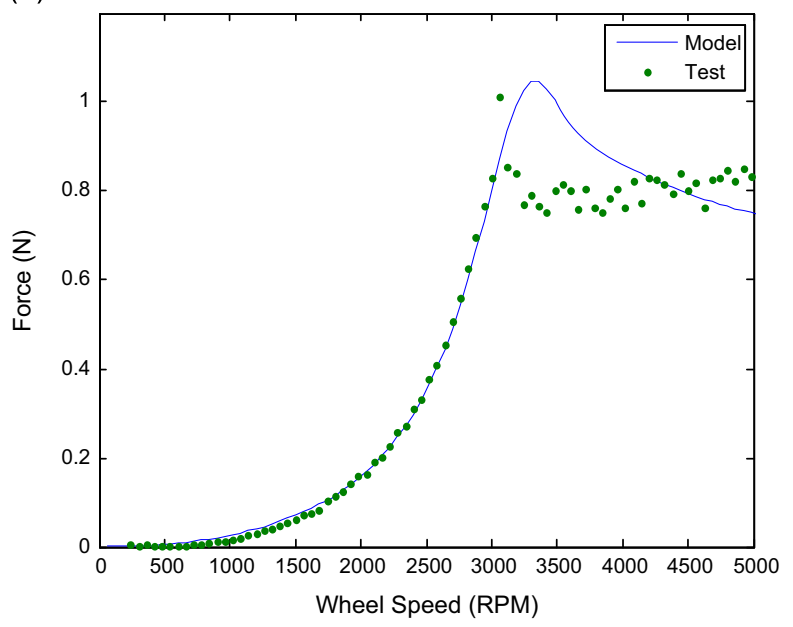

(b)

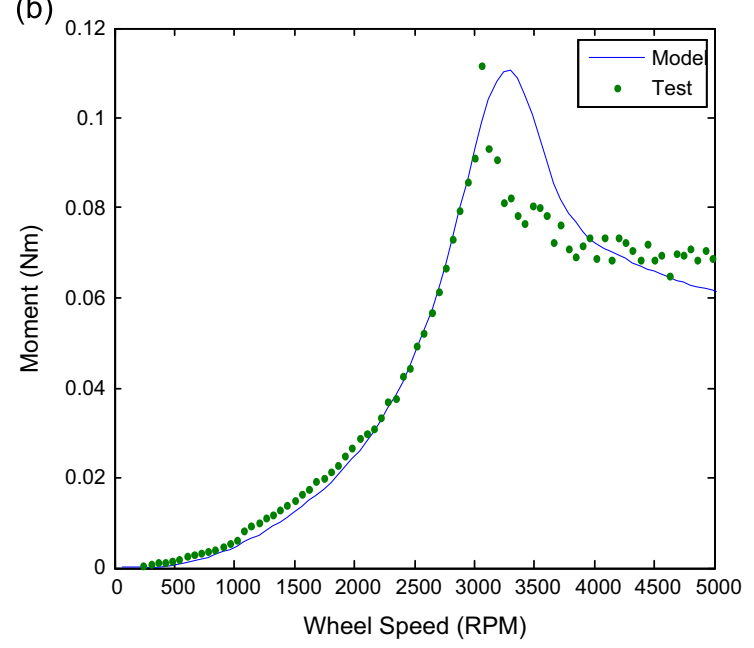

(c)

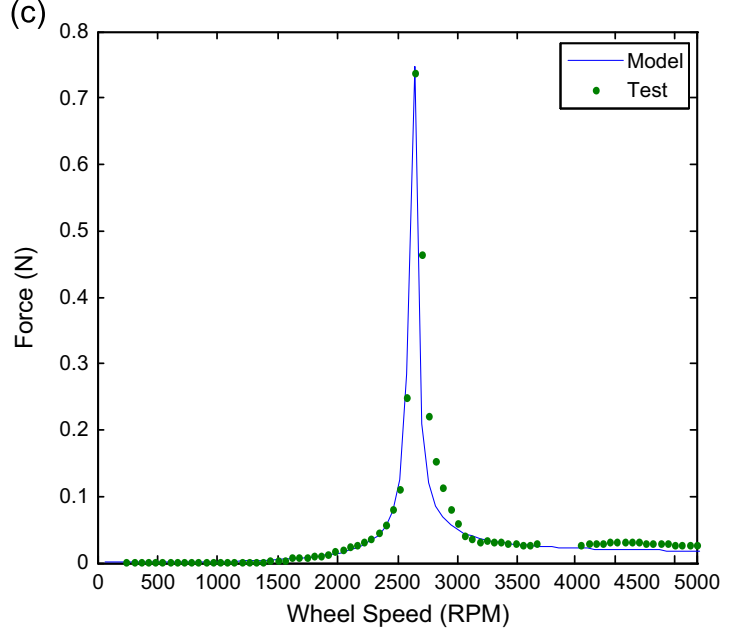

Fig. 5. Comparisons of $\mathrm{H} 1$ responses of WA-seismic mass coupled system: (a) $F_{y i}$; (b) $M_{x i}$ and (c) $F_{z i}$. 
results in in-plane dofs due to nonlinearity. The decreasing trends after resonances also follow the test results reasonably well in each dof. $F_{z i}$ is simulated well in the complete speed band as nonlinearity is insignificant here.

For conventional WA designs (i.e. flywheel rigidly supported), the primary resonances are usually beyond the typical maximum spin speed, e.g. $5000 \mathrm{rev} / \mathrm{min}$. The coupled microvibration model introduced in this article is also capable of simulating their microvibrations. The modeling method introduced in this section has been applied to another WA with conventional design configurations and successfully simulated its microvibrations in the hard-mounted boundary condition (see [24] for detail). The coupled microvibration model presented in this section is also used as the basis for the WA dynamic accelerance derivations shown in Section 3.3.

\section{Model development of WA accelerances}

\subsection{Introduction}

Generally, in a WA-structure coupled system, the accelerances of the two bodies at the location where they are connected (i.e. the driving point) are particularly important in the coupled microvibration analysis. The relationship between WA-induced microvibrations and the receiver responses can be expressed mathematically as following [17]:

$$
\boldsymbol{\Phi}_{Z Z \_ \text {receiver }}(\omega, \Omega)=\mathbf{G}_{Z F}(\omega) \mathbf{G}_{f}(\omega, \Omega) \mathbf{\Phi}_{F F_{\text {grounded }}}(\omega, \Omega) \mathbf{G}_{f}^{H}(\omega, \Omega) \mathbf{G}_{Z F}^{H}(\omega)
$$

where the transfer matrix, $\mathbf{G}_{f}(\omega, \Omega)$, is

$$
\mathbf{G}_{f}(\omega, \Omega)=\left[\mathbf{I}+\mathbf{A}_{w}^{-1}(\omega, \Omega) \mathbf{A}_{s}(\omega)\right]^{-1}
$$

In Eq. (4), $\boldsymbol{\Phi}_{F F_{-} \text {grounded }}$ is a $6 \times 6$ matrix of WA-induced loads in hard-mounted condition (i.e. power spectral densities (PSDs) and cross-spectral densities (CSDs) of forces and moments), which act as the inputs; $\boldsymbol{\Phi}_{Z Z \text { receiver }}$ is a $6 \times 6$ matrix of receiver responses also expressed in terms of PSDs and CSDs, which act as the outputs. $\mathbf{G}_{\mathrm{ZF}}(\omega)$ is a $6 \times 6$ transfer matrix from the driving point to the receiver. The transformation matrix, $\mathbf{G}_{f}(\omega, \Omega)$, is a $6 \times 6$ matrix and includes the WA driving point accelerance, $\mathbf{A}_{w}(\omega, \Omega)$, which is both frequency-dependent and speed-dependent, and the structure driving point accelerance, $\mathbf{A}_{s}(\omega)$, which depends only on frequency. Finally $\mathbf{I}$ is a $6 \times 6$ unit matrix and "H" indicates the Hermitian or complex conjugate transpose of a square matrix.

The focus of this article is the relationship between the WA hard-mounted microvibrations, $\boldsymbol{\Phi}_{F F_{-} \text {grounded }}$, and the coupled microvibrations, $\boldsymbol{\Phi}_{F F_{-} \text {coupled, }}$ in Eq. (4). This can be expressed as

$$
\boldsymbol{\Phi}_{F F_{-} \text {coupled }}(\omega, \Omega)=\mathbf{G}_{f}(\omega, \Omega) \mathbf{\Phi}_{F F_{-} \text {grounded }}(\omega, \Omega) \mathbf{G}_{f}^{H}(\omega, \Omega)
$$

In this case it is clearly that $\mathbf{G}_{f}(\omega, \Omega)$ acts as a transfer matrix between the two types of microvibrations. This matrix is sometime referred to as the "load filter", which corrects or modifies the hard-mounted microvibrations to the desired coupled microvibrations. $\boldsymbol{\Phi}_{F F}$ coupled can then be applied directly to the satellite structure transfer matrix, $\mathbf{G}_{Z F}(\omega)$, to predict the receiver responses from Eq. (4).

In practice, $\boldsymbol{\Phi}_{F F_{-} \text {grounded }}$ is usually measured by a grounded Kistler table or load cell. While $\mathbf{A}_{s}(\omega)$ can be obtained from the satellite FE model, which is generally reliable; the difficulty is in obtaining $\mathbf{A}_{w}(\omega, \Omega)$. In this article, experimental and analytical methods are developed to obtain the WA driving point accelerances with and without gyroscopic effects, and used respectively to predict the coupled microvibrations, $\boldsymbol{\Phi}_{F F_{-} \text {coupled }}$.

\subsection{WA static accelerances}

In this section, WA driving point accelerances in a static condition, i.e. with the flywheel not spinning (or static accelerances for simplicity), are obtained. Analytically, static accelerances are obtained using frequency response analysis in NASTRAN on a WA FE model created. Experimentally, an accelerance measurement system is designed to measure the WA (static and dynamic) accelerances by using common accelerometers and force sensors. WA static accelerances obtained from the two methods are compared and both methods are validated.

\subsubsection{The experimental method}

In general, the complete representation of the $6 \times 6$ WA accelerance matrix can be shown as following:

$$
\left[\begin{array}{l}
\ddot{x} \\
\ddot{y} \\
\ddot{z} \\
\ddot{\theta} \\
\ddot{\varphi} \\
\ddot{\psi}
\end{array}\right]=\left[\begin{array}{llllll}
\underline{A}_{w 11} & A_{w 12} & A_{w 13} & A_{w 14} & \underline{A}_{w 15} & A_{w 16} \\
A_{w 21} & \underline{A}_{w 22} & A_{w 23} & \underline{A}_{w 24} & A_{w 25} & A_{w 26} \\
A_{w 31} & A_{w 32} & \underbrace{}_{w 33} & A_{w 34} & A_{w 35} & A_{w 36} \\
A_{w 41} & \underline{A}_{w 42} & A_{w 43} & \underline{A}_{w 44} & A_{w 45} & A_{w 46} \\
\underline{A}_{w 51} & A_{w 52} & A_{w 53} & A_{w 54} & \underline{A}_{w 55} & A_{w 56} \\
A_{w 61} & A_{w 62} & A_{w 63} & A_{w 64} & A_{w 65} & A_{w 66}
\end{array}\right]\left[\begin{array}{l}
F_{x} \\
F_{y} \\
F_{z} \\
M_{x} \\
M_{y} \\
M_{z}
\end{array}\right]
$$


(a)

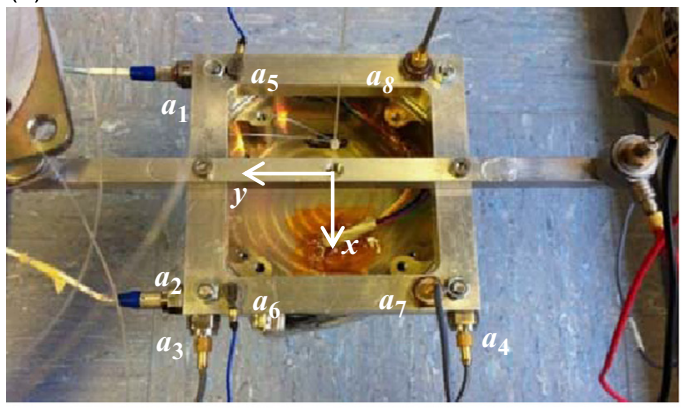

(c)

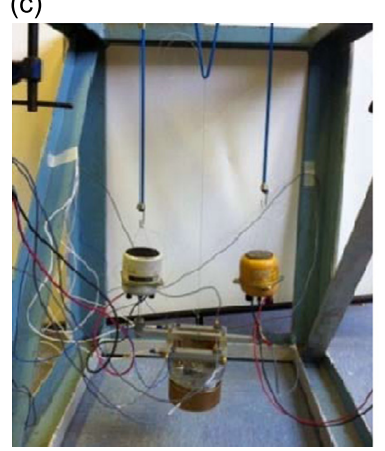

(b)

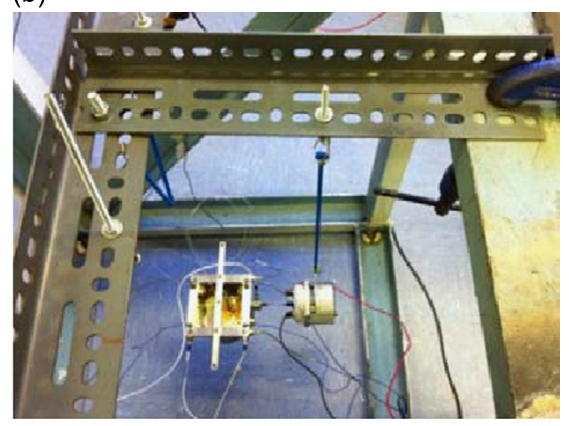

(d)

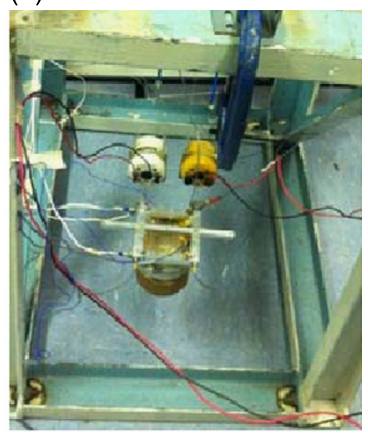

Fig. 6. WA accelerance tests: (a) accelerometer positions; (b) $A_{w 11}$ and $A_{w 51}$ test; (c) $A_{w 33}$ and $A_{w 44}$ test; and (d) $A_{w 66}$ test.

Previous studies in [21-23] have shown that the diagonal elements (or the direct driving point accelerances): $A_{w 11}, A_{w 22}$, $A_{w 33}, A_{w 44}, A_{w 55}, A_{w 66}$ are the most significant. The four cross-dof accelerance elements: $A_{w 15}, A_{w 24}, A_{w 51}$ and $A_{w 42}$ are also significant, especially when CSDs in inputs are considered. These elements are underlined in Eq. (7). Other off-diagonal elements are not considered significant as they generally tend to be zero, and are thus ignored. Since the matrix is symmetric about its diagonal due to WA axisymmetry, the four underlined cross-dof elements are the same. In addition, diagonal elements $A_{w 11}$ and $A_{w 22}$ are the same and so are $A_{w 44}$ and $A_{w 55}$. For simplicity, five elements in the accelerance matrix are chosen for the coupled microvibration analysis in this article, they are identified by circles in Eq. (7).

Three independent tests were carried out to find the five elements mentioned in the WA accelerance matrix. Test setups are shown in Fig. 6(b)-(d) correspondingly. A mounting bracket is designed and rigidly connected to the WA base interface. Accelerometers on the mounting bracket are shown in Fig. 6(a).

In each test, the WA was hung "free-free" using elastic cords from a rigid steel frame. The suspension was designed such that its natural frequency was less than $1 \mathrm{~Hz}$ in any of the six dofs. Input forces and/or moments were applied by one or two mini-shakers and measured by force sensors inserted between the stingers and mounting bracket. Response accelerations were measured using eight accelerometers (see Fig. 6(a)). The mounting bracket was designed such that the system modal frequency in any of the six dofs is far beyond the maximum frequency of interest.

The linear and angular response accelerations at the driving point can be calculated using the following equations:

$$
\begin{gathered}
\ddot{x}=\frac{1}{2}\left(a_{3}+a_{4}\right) \\
\ddot{y}=\frac{1}{2}\left(a_{1}+a_{2}\right) \\
\ddot{z}=-\frac{1}{4}\left(a_{5}+a_{6}+a_{7}+a_{8}\right) \\
\ddot{\theta}=\frac{1}{2 d_{67}}\left(a_{7}+a_{8}-a_{5}-a_{6}\right) \\
\ddot{\varphi}=\frac{1}{2 d_{56}}\left(a_{6}+a_{7}-a_{5}-a_{8}\right) \\
\ddot{\psi}=\frac{1}{d_{34}}\left(a_{4}-a_{3}\right)
\end{gathered}
$$

where $d_{67}, d_{56}$ and $d_{34}$ are distances between each pair of accelerometers in the coordinate system (see Fig. 6(a)). 
Sine-sweep tests were performed from 10 to $500 \mathrm{~Hz}$ and all signals were sampled at $2048 \mathrm{~Hz}$. Each test is briefly described below:

- Fig. 6(b) depicts the setup for $A_{w 11}$ and $A_{w 51}$ tests. The WA was excited in positive $x$-axis using one mini-shaker. Linear and angular accelerations at the driving point were calculated using Eqs. (8) and (12) respectively.

- Fig. 6(c) depicts the setup for $A_{w 33}$ and $A_{w 44}$ tests. For $A_{w 33}$ test, two mini-shakers were controlled in in-phase. Linear accelerations at the driving point were calculated using Eq. (10). For $A_{w 44}$ test, two mini-shakers were controlled in antiphase and a moment at the driving point was produced.

- Fig. 6(d) depicts the setup for $A_{w 66}$ test. The two mini-shakers were attached in positive $x$-axis and controlled in antiphase and a moment about $z$-axis at the driving point was produced.

\subsubsection{The analytical method and results validation}

The $6 \times 6$ WA static accelerance matrix was obtained from the frequency response analysis of its FE model performed in NASTRAN. In frequency response analysis, a unit force or moment was applied at the driving point of the "free-free" FE model in one of the six dofs. Accelerations were calculated at the same point in six dofs, thus a $6 \times 1$ vector of accelerances at the driving point was obtained. This process was repeated for the other five dofs and a $6 \times 6$ accelerance matrix was ultimately obtained.

The WA static accelerance elements, $A_{w 11}, A_{w 33}, A_{w 44}$ and $A_{w 66}$, from the FE model and tests are compared. They are shown in Fig. 7 respectively.
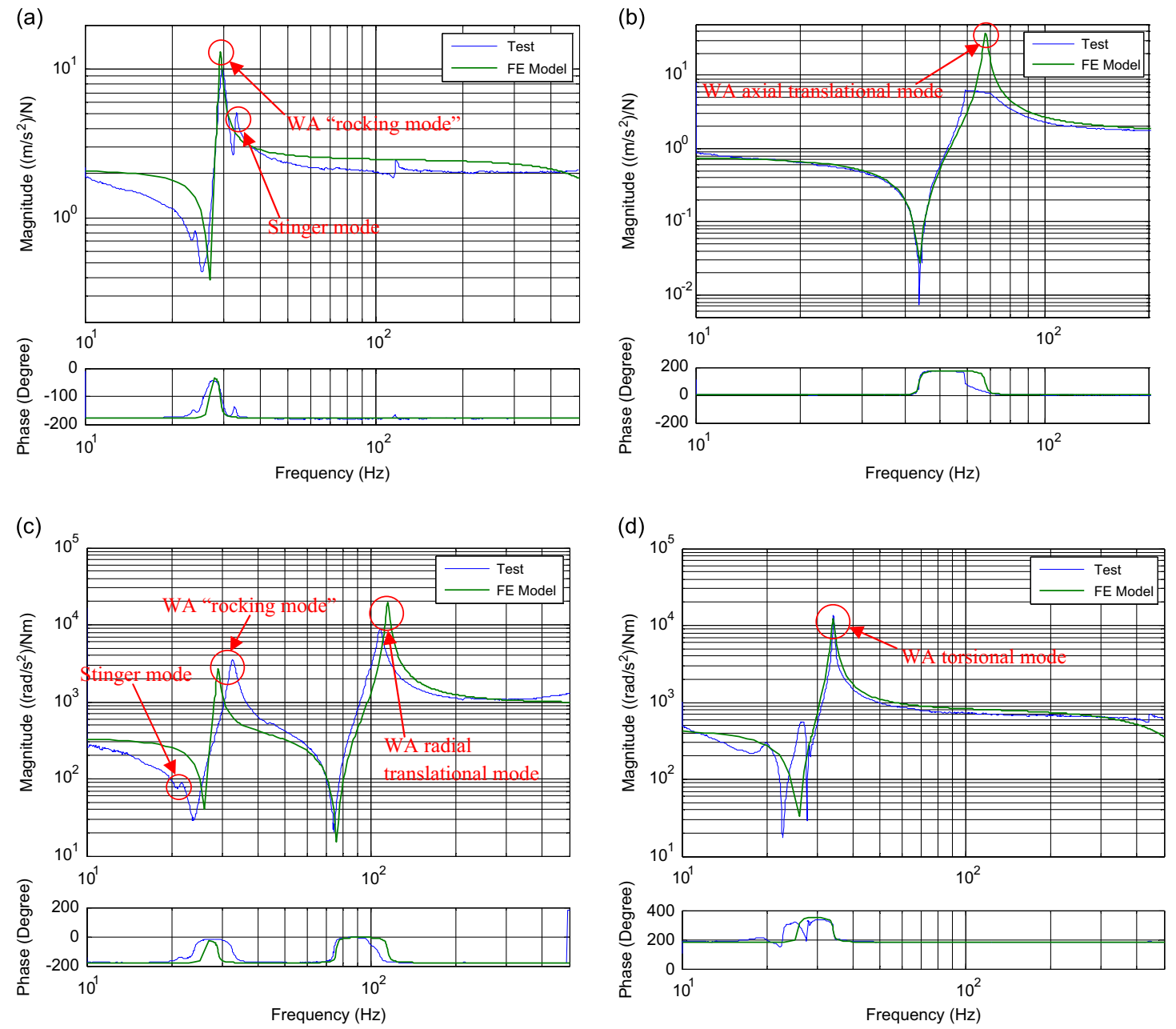

Fig. 7. Comparisons of WA direct driving point static accelerances: (a) in-plane translational dof $\left(A_{w 11}\right)$; (b) axial translational dof $\left(A_{w 33}\right)$; (c) in-plane rotational dof $\left(A_{w 44}\right)$; and (d) torsional dof $\left(A_{w 66}\right)$. 


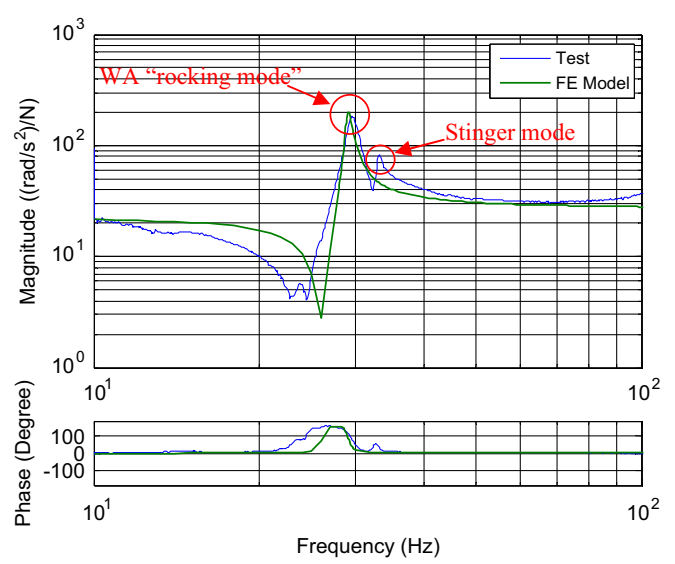

Fig. 8. Comparisons of WA cross-dof driving point static accelerance $\left(A_{w 51}\right)$.

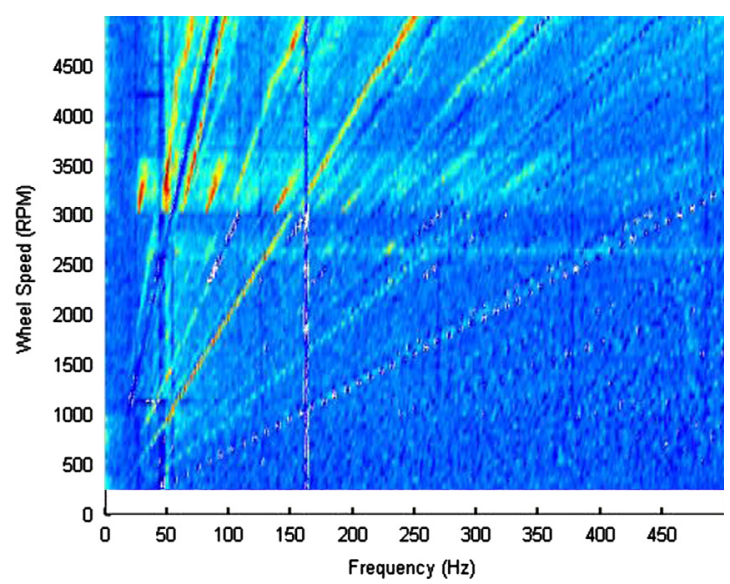

Fig. 9. "Empirical load filter"-axial translational dof.

In Fig. 7, results from the two methods have matched well between each other in both magnitude and phase in the frequency band considered. Note in the test results, a small spike between 25 and $35 \mathrm{~Hz}$ was measured in each in-plane dofs. Further investigations showed that this spike was due to the influences of stingers and it could be avoided by extending them. The updated accelerance test results are shown in Fig. 10 in Section 3.3.1. Generally, the mode of stinger would not significantly influence the measured accelerances as they are hardly noticed, even in the plot on logarithmic scale, such as those in Fig. 7.

Comparisons of WA static accelerance element, $A_{w 51}$, are shown in Fig. 8.

Two results have again matched closely for $A_{w 51}$. In general, the five static accelerances shown above can be used to represent the WA accelerance matrix, $\mathbf{A}_{w}(\omega)$, in static condition. The coupled microvibrations can therefore be obtained using Eq. (6) in the traditional sense.

In summary, the results from Figs. 7 and 8 indicate that the measurement method introduced in this article is able to obtain the WA driving point accelerances at static condition with reasonable accuracy. However, it must be stressed that the use of WA static accelerances is flawed if the analysis is for a spinning WA, as it does not take into account gyroscopic effects. Alternatively, the coupled microvibrations predicted with static accelerances from Eq. (6) are only accurate when the WA is static or at very low spin speeds (say up to $1000 \mathrm{rev} / \mathrm{min}$ ). It is thus necessary to develop a method to include the gyroscopic effects in the WA accelerances for analysis in broadband speeds.

\subsection{WA dynamic accelerance}

\subsubsection{Gyroscopic effects in accelerances}

The differences between the hard-mounted and coupled microvibrations can be directly seen from the microvibration test results. In this case, $\mathbf{G}_{f}(\omega, \Omega)$ in Eq. (6) is known as the "empirical load filter" if test results $\boldsymbol{\Phi}_{F F_{-} \text {grounded }}$ and $\boldsymbol{\Phi}_{F F_{-} \text {coupled }}$ 
are used. The general form of $\boldsymbol{\Phi}_{F F_{-} \text {grounded }}$ and $\boldsymbol{\Phi}_{F F_{-} \text {coupled }}$ is

$$
\boldsymbol{\Phi}_{F F}(\omega, \Omega)=\left[\begin{array}{llllll}
\Phi_{F_{x} F_{x}} & \Phi_{F_{x} F_{y}} & \Phi_{F_{x} F_{z}} & \Phi_{F_{x} M_{x}} & \Phi_{F_{x} M_{y}} & \Phi_{F_{x} M_{z}} \\
\Phi_{F_{y} F_{x}} & \Phi_{F_{y} F_{y}} & \Phi_{F_{y} F_{z}} & \Phi_{F_{y} M_{x}} & \Phi_{F_{y} M_{y}} & \Phi_{F_{y} M_{z}} \\
\Phi_{F_{z} F_{x}} & \Phi_{F_{z} F_{y}} & \Phi_{F_{z} F_{z}} & \Phi_{F_{z} M_{x}} & \Phi_{F_{z} M_{y}} & \Phi_{F_{z} M_{z}} \\
\Phi_{M_{x} F_{x}} & \Phi_{M_{x} F_{y}} & \Phi_{M_{x} F_{z}} & \Phi_{M_{x} M_{x}} & \Phi_{M_{x} M_{y}} & \Phi_{M_{x} M_{z}} \\
\Phi_{M_{y} F_{x}} & \Phi_{M_{y} F_{y}} & \Phi_{M_{y} F_{z}} & \Phi_{M_{y} M_{x}} & \Phi_{M_{y} M_{y}} & \Phi_{M_{y} M_{z}} \\
\Phi_{M_{z} F_{x}} & \Phi_{M_{z} F_{y}} & \Phi_{M_{z} F_{z}} & \Phi_{M_{z} M_{x}} & \Phi_{M_{z} M_{y}} & \Phi_{M_{z} M_{z}}
\end{array}\right]
$$

Traditionally, the coupled microvibration spectra $\left(\boldsymbol{\Phi}_{F F_{-} \text {coupled }}\right)$ can be measured directly by inserting a load cell at the interface between the WA and the supporting structure. On the other hand, the hard-mounted microvibrations from WA ( $\boldsymbol{\Phi}_{F F_{\text {g grounded }}}$ ) can be measured, for example by using a grounded Kistler table or load cell. Microvibrations measured in both cases can be written as $6 \times 6$ spectral density matrices. In order to study the gyroscopic effects in WA accelerances, microvibrations measured from the WA-seismic mass coupled system (shown in Section 2) are used. Note that in this system, forces and moments obtained at the interface between the WA and seismic mass directly represent $\boldsymbol{\Phi}_{\text {FF_coupled }}$. The WA hard-mounted microvibrations are taken from a previous study in [19] but are not shown in this article.

For simplicity, only the microvibrations in the independent axial translational dof (i.e. $\Phi_{F z F z}$ ) are shown. The root square of the ratio of the hard-mounted and coupled microvibrations in axial translational dof is directly the "empirical load filter" of that dof. The calculated results in the speed band are plotted in Fig. 9.

From Fig. 9 two important characteristics of WA dynamic accelerances can be seen:

- Harmonic responses are almost eliminated in each dof. Since amplitudes of the measured harmonic responses of hardmounted and coupled microvibrations are very close to each other at each frequency and each speed, their ratio is close
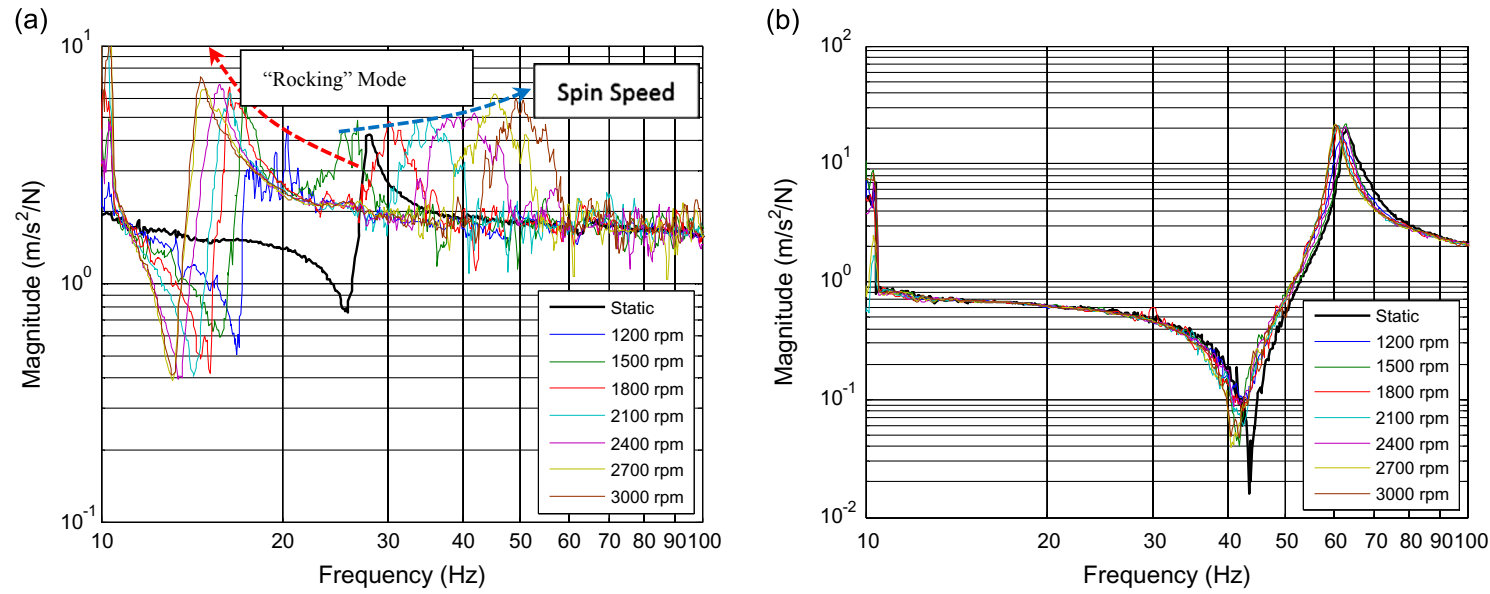

(c)

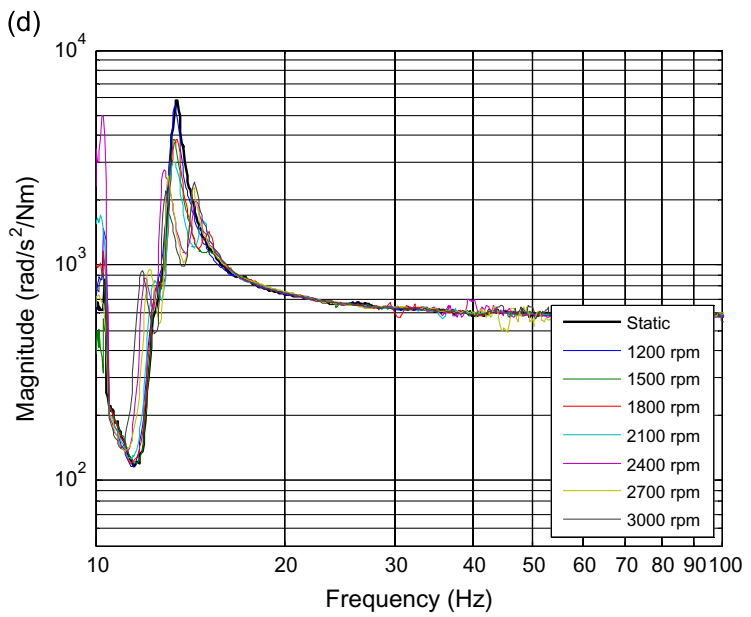

Fig. 10. WA driving point dynamic accelerances at selected speeds: (a) in-plane translational dof $\left(A_{w 11}\right)$; (b) axial translational dof $\left(A_{w 33}\right)$; (c) in-plane rotational dof $\left(A_{w 44}\right)$; and (d) torsional dof $\left(A_{w 66}\right)$. 
to unity. This observation indicates harmonics produced by the spinning WA are not part of the WA accelerances, which is expected.

- Nonlinearity also appears in the WA accelerance at high spin speeds (above $3060 \mathrm{rev} / \mathrm{min}$ in the figure). Though, in this article, the predicted coupled microvibrations at high speeds cannot be used for data comparison as the influences of nonlinearity are not modeled, and thus are not included in accelerance simulations.

Note the experimental method to obtain the "empirical load filter" introduced in this section cannot be used to predict accelerances in in-plane dofs due to non-zero off-diagonal elements which cannot be ignored. On the other hand, the fully populated "empirical load filters" are very difficult or impossible to obtain experimentally.

The WA dynamic accelerances were measured at selected speeds (from 1200 to $3000 \mathrm{rev} / \mathrm{min}$ with step increase at every $300 \mathrm{rev} / \mathrm{min}$ ). In this case, the flywheel spins while a sine-sweep was performed and accelerations were measured. Dynamic accelerances, $A_{w 11}, A_{w 33}, A_{w 44}$ and $A_{w 66}$, at the selected speeds are plotted in Fig. 10 respectively. Note only magnitudes are plotted and signals are smoothed to reduce the broadband noise.

In this case, influences of gyroscopic effects are clearly shown in the measured dynamic accelerances. For example, in Fig. 10(a) and (c), the WA "rocking" mode (around $27 \mathrm{~Hz}$ ) has appeared and is speed-dependent as expected, thus $A_{w 11}$ and $A_{w 44}$ are also speed-dependent. In particular, it is noticed that the "rocking" mode decreases as speed increases (see arrows in Fig. 10(a) and (c)). It is verified that this decreasing trend follows the backward whirl (BW1) of the "rocking" mode (see Fig. 4(a) for reference). Other structural modes such as the in-plane translational mode (around $100 \mathrm{~Hz}$ in Fig. 10(a) and (c)), the axial translational mode (around $63 \mathrm{~Hz}$ in Fig. 10(b)) and the torque mode (around $14 \mathrm{~Hz}$ in Fig. 10(d)) do not show significant changes with the spin speed. Another mode in the test results at approximately $10 \mathrm{~Hz}$, due to the overall natural frequency of the flywheel-base system, it is further introduced in Section 3.3.2.1.

It should be mentioned that the measurement method introduced above is not entirely practical for several reasons, such as the fact that it requires theoretical corrections. The accelerations measured during the tests are the sum of the responses to the external inputs provided by the shaker, but unfortunately they also include influences due to flywheel mass imbalances, etc., which should not influence the accelerance in theory. In other words, harmonics are also included in the test results (see arrows in Fig. 10(a) and (c)), although they should not be (see Fig. 9 for comparison). Meanwhile, the direct measurement of dynamic accelerances requires WA spins at every speed in the speed band with excitations being applied and data recorded. This process is extremely time-consuming and results are not always reliable.

In conclusion, the two experimental methods introduced in this section are not practical for the coupled WA microvibration analysis. However, the results from both methods have clearly shown the differences between static and dynamic accelerances for a WA. Gyroscopic effects due to the spinning flywheel must be included in WA accelerance modeling, especially at high spin speeds. So far the most efficient approach is to model them analytically using a validated WA microvibration model. A method to calculate the WA dynamic accelerances is introduced in the next section.

\subsubsection{The analytical method}

The accelerance of a mechanical system is a property of the system, but the driving point accelerances depend on the locations of forces and accelerations taken on the system. The "driving point" for a typical WA is at its base interface, while the supporting structure shares the same interface as its driving point. In literature works, analytical expressions of the dynamic accelerances are derived from WA microvibration model in hard-mounted boundary condition and considering only the flywheel properties in the model, while stiffness and damping of the suspension system are excluded. Therefore, WA dynamic accelerances calculated from such models are constant values in frequency domain in all six dofs at any speed (i.e. either the flywheel mass or moment of inertias). These analytical results contradict the test results, as shown in Fig. 10. For example, the static accelerances are clearly non-constant in frequency domain due to the presence of the internal dynamics and resonances of the WA. This is due to the model available in the literature not including the WA internal modes, therefore it produces inaccurate driving point dynamic accelerances and the coupled microvibrations predicted show inconsistencies. For this reason, a new method to calculate the WA driving point dynamic accelerances is developed.

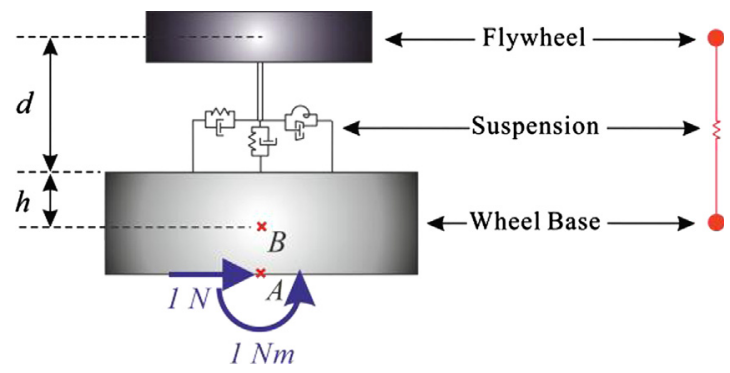

Fig. 11. WA model in "free-free" boundary condition. 
3.3.2.1. WA dynamic accelerance model. The driving point dynamic accelerances of a WA are obtained when the WA is in "free-free" boundary condition. The WA model in "free-free" boundary condition can be depicted as following:

With reference to Fig. 11, the WA base can be modeled as a rigid point mass with inertias at its CoM. The EoM of the flywheel-base system can be derived following the same approach as for the WA-seismic mass coupled system. In this case, the properties of the WA base are used instead of those of the seismic mass. In general, it is written in frequency domain as

$$
\mathbf{M}_{s s} \ddot{\mathbf{q}}_{s s}+\left(\mathbf{C}_{s s}+\mathbf{G}_{s s}\right) \dot{\mathbf{q}}_{s s}+\mathbf{K}_{s s} \mathbf{q}_{s s}=\mathbf{F}_{s s}
$$

where subscript "ss" indicates the flywheel-base system.

Assuming zero initial conditions, the relationship between $\ddot{\mathbf{q}}_{s s}, \dot{\mathbf{q}}_{s s}$ and $\mathbf{q}_{s s}$ is

$$
\ddot{\mathbf{q}}_{s s}=\mathrm{j} \omega \dot{\mathbf{q}}_{s s}=-\omega^{2} \mathbf{q}_{s s}
$$

where $\mathrm{j}$ is the imaginary unit. Therefore Eq. (15) can also be written as

$$
\mathbf{M}_{s s} \ddot{\mathbf{q}}_{s s}+\left(\mathbf{C}_{s s}+\mathbf{G}_{s s}\right) \frac{1}{\mathrm{j} \omega} \ddot{\mathbf{q}}_{s s}-\mathbf{K}_{s s} \frac{1}{\omega^{2}} \ddot{\mathbf{q}}_{s s}=\mathbf{F}_{s s}
$$

and the WA dynamic accelerances, $\mathbf{A}_{w}(\omega, \Omega)$, can be expressed from Eq. (17) as

$$
\mathbf{A}_{w}(\omega, \Omega)=\left[\mathbf{M}_{s s}+\left(\mathbf{C}_{s s}+\mathbf{G}_{s s}\right) \frac{1}{\mathrm{j} \omega}-\mathbf{K}_{s s} \frac{1}{\omega^{2}}\right]^{-1} \mathbf{F}_{s s}
$$

Eq. (18) is both frequency-dependent $(\omega)$ and speed-dependent $(\Omega)$. The mass matrix, $\mathbf{M}_{s s}$, damping matrix, $\mathbf{C}_{s s}$, gyroscopic matrix, $\mathbf{G}_{s s}$, and stiffness matrix, $\mathbf{K}_{s s}$, include those of the flywheel and base. $\mathbf{F}_{s s}$ is the forcing vector, $\ddot{\mathbf{q}}_{s s}$ is the response vector. Elements in the matrices and vectors are taken at the CoM of the flywheel and base respectively. They are similar to those given in Appendix A but by replacing seismic mass properties with those of WA base, and are thus not presented.

The definition of the driving point accelerance states that excitations and responses must be taken at the same point. In the flywheel-base system in Fig. 11, the only microvibrations produced are by "defects" of the WA internal components and are idealized at the flywheel CoM as harmonic excitations. In order to find the driving point accelerances, unit force or moment can be applied at the base interface (point A on the model in Fig. 11) in Eq. (17) one component at a time, and $\ddot{\mathbf{q}}_{s s}$, which corresponds to the related column in $\mathbf{A}_{w}(\omega, \Omega)$ is calculated.

It is also worth mentioning that $\mathbf{A}_{w}(\omega, \Omega)$ is a $10 \times 10$ matrix at each speed (see Appendix B for its general form). If the unit load method is used to find accelerance at the base CoM, only the 25 elements from the WA base are eventually required. These 25 elements form a fully populated matrix (with the exception of the independent axial translational dof), thus the driving point accelerances of the flywheel-base system is a $5 \times 5$ symmetric matrix with non-zero off-diagonal elements.

Overall, with respect to the two experimental methods in Section 3.3.1, the analytical method has the advantage that is able to provide the fully populated accelerance matrix.

3.3.2.2. The simulated WA dynamic accelerances. Eq. (18) is simulated in the frequency band between $1 \mathrm{and} 500 \mathrm{~Hz}$ and speed band between 1 and $5000 \mathrm{rev} / \mathrm{min}$. Fig. 12 shows comparisons of magnitudes of $A_{w 33}$ and $A_{w 44}$ obtained from analytical expressions and from test results. The test results at static and $2400 \mathrm{rev} / \mathrm{min}$ are shown as examples.

In Fig. 12(a), analytical and test results match very well for $A_{w 33}$, the accelerance simply reveals the speed-independent axial translational mode. The $A_{w 44}$ in Fig. 12(b) on the other hand, shows small dissimilarities. The "rocking" mode due to gyroscopic effects is predicted and the general trend follows the test results reasonably well. The speed-independent mode

(a)

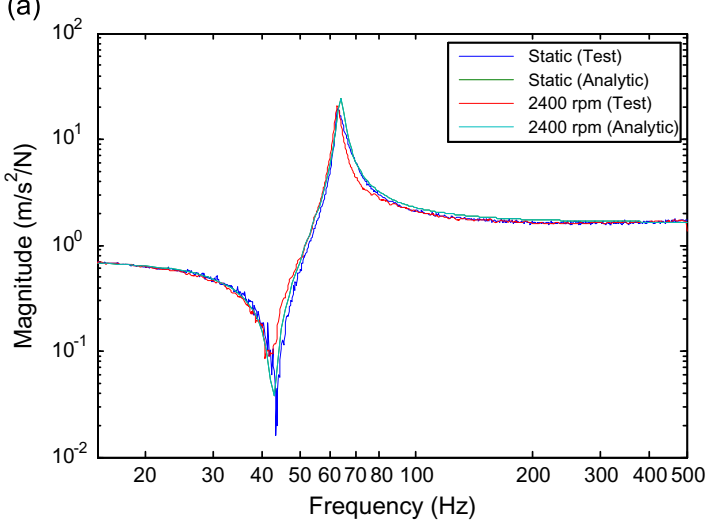

(b)

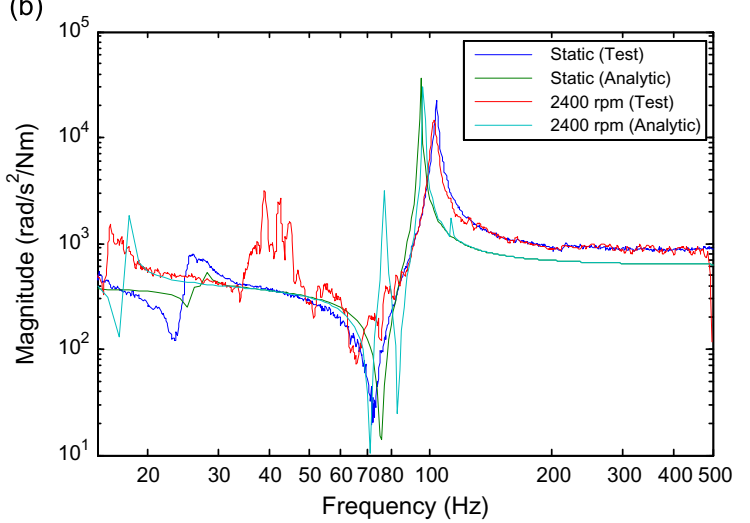

Fig. 12. Experimental and analytical WA driving point accelerance comparisons: (a) axial translational dof $\left(A_{w 33}\right)$ and (b) in-plane rotational dof $\left(A_{w 44}\right)$. 
(a)

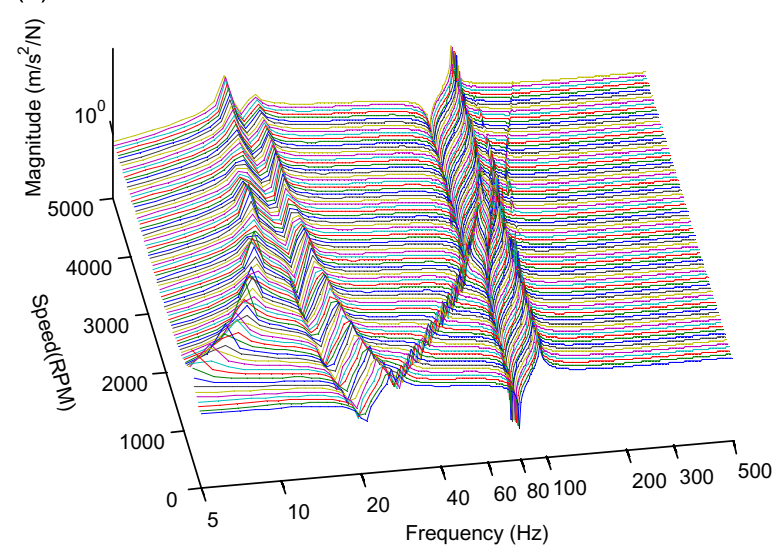

(c)

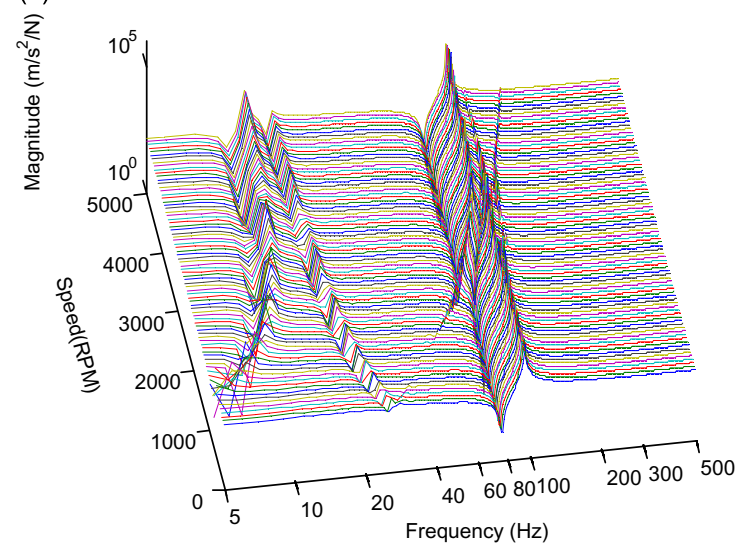

(b)

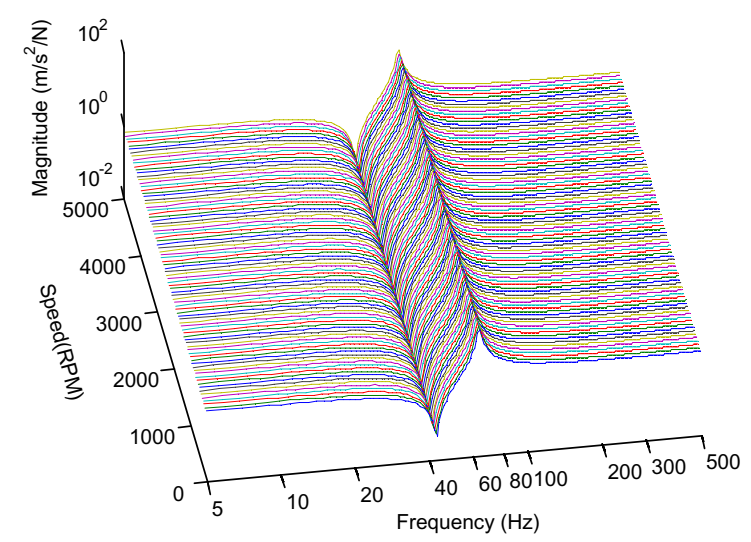

(d)

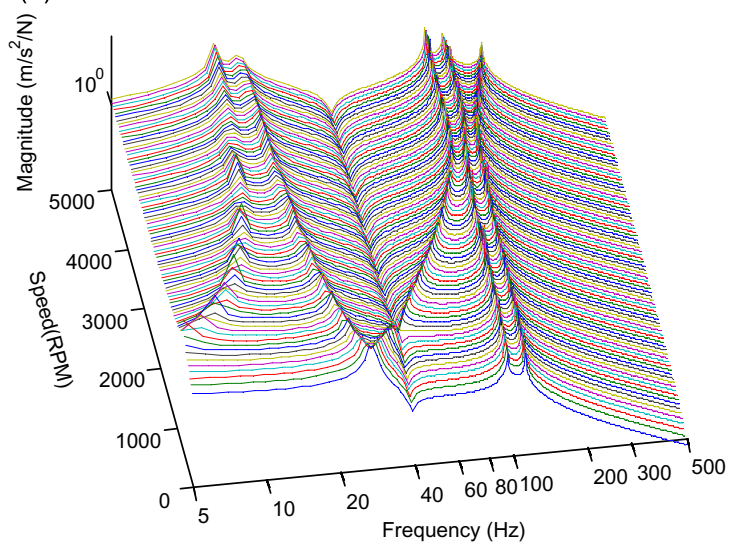

Fig. 13. Simulated results of the WA driving point dynamic accelerances. (a) In-plane translational dof $\left(A_{w 11}\right)$; (b) Axial translational dof $\left(A_{w 33}\right)$; (c) in-plane rotational dof $\left(A_{w 44}\right)$; and (d) cross-dof $\left(A_{w 14}\right)$.
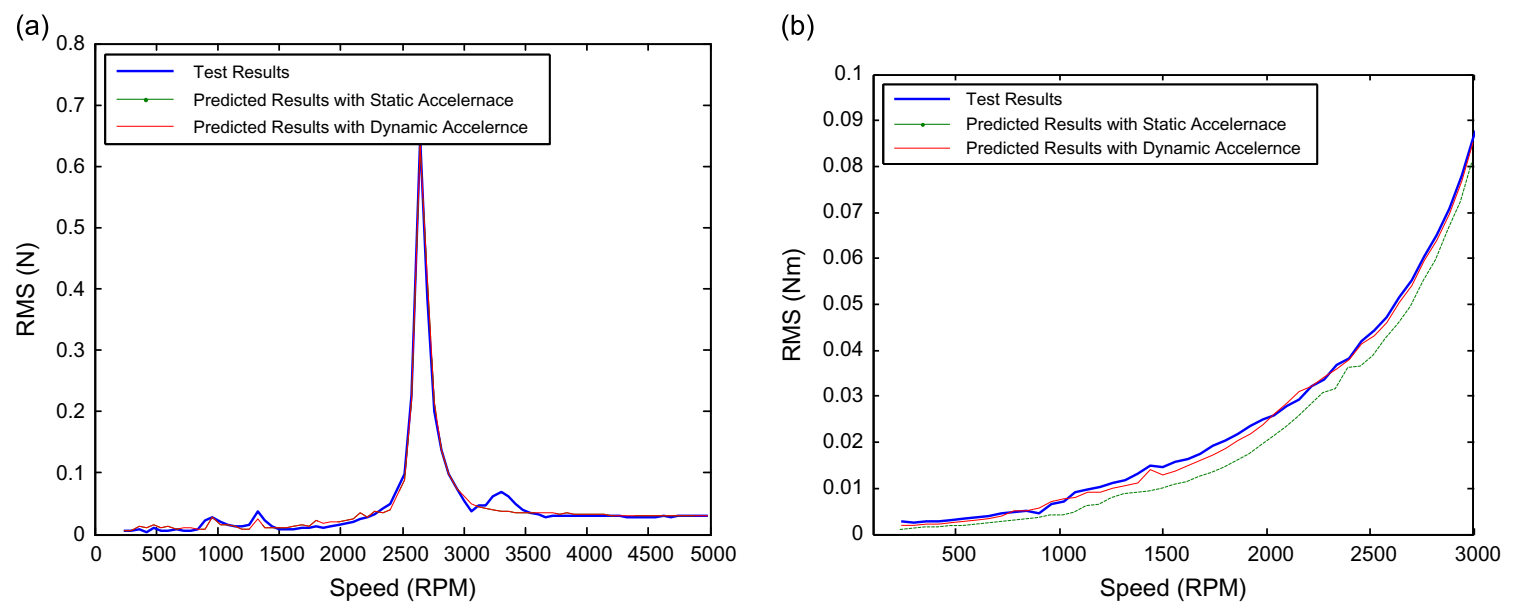

Fig. 14. Comparisons of coupled microvibrations predicted with static accelerances, dynamic accelerances and test results: (a) $\Phi_{C F Z F z}$ and (b) $\Phi_{C M y M y}$ (plotted up to $3000 \mathrm{rev} / \mathrm{min}$ as data at higher spin speeds are not reliable due to nonlinearity).

around $100 \mathrm{~Hz}$ in $A_{w 44}$ is also well matched. The minor differences may due to influences of the mounting bracket which are not considered in the WA microvibration model in Fig. 11. In general, Eq. (18) can be used to calculate the WA driving point dynamic accelerances with reasonable accuracy. 
Analytical results of the three direct driving point dynamic accelerances, $A_{w 11}, A_{w 33}$ and $A_{w 44}$, and one selected cross-dof driving point dynamic accelerance, $A_{w 14}$, were simulated in the speed band. Results are shown in the following figure (magnitudes only).

All speed-dependent and/or independent modes of the WA are clearly seen in each plot. In Fig. 13(a) and (c), the forward and backward whirls of the "rocking" mode are represented in in-plane dofs, but the backward whirl appears more significant. This can be interpreted as when the speed increases, the gyroscopic effects mainly shift influence of the "rocking" mode to lower frequencies. This observation matches the test results shown in Fig. 10. It is also observed that the speed-independent mode around $100 \mathrm{~Hz}$ in Fig. 13(a) and (c) are not always constant. They are in fact a composition of the forward whirl (above $2700 \mathrm{rev} / \mathrm{min}$ ) and the in-plane translational mode (below $2700 \mathrm{rev} / \mathrm{min}$ ). Since they appear almost constant in the two speed bands, they are together considered as the in-plane translational mode of the WA.

Another speed-dependent mode up to about $10 \mathrm{~Hz}$ is due to the overall natural frequency of the flywheel-base system. This mode is also influenced by gyroscopic effects as also seen in the test results in Fig. 10. However it is not the main concern in the frequency band of interest as it is limited up to $10 \mathrm{~Hz}$, and can be thus ignored. The axial translational mode shown in Fig. 13(b) is decoupled from those of in-plane dofs and constant in the speed band; $A_{w 14}$ in Fig. 13(d) includes all structural modes in in-plane dofs, both results are as expected.

\subsubsection{The coupled microvibrations}

The driving point accelerance of the mounting structure, $\mathbf{A}_{s}(\omega)$, is usually obtained from its FE model. Since in this case the seismic mass is a rigid body in the frequency band of interest, all elements in $\mathbf{A}_{s}(\omega)$ are constant values in the speed band and are not shown here.

The coupled microvibrations, $\boldsymbol{\Phi}_{F F-c o u p l e d}$, of the WA-seismic mass system are predicted from two methods, with WA static accelerances and dynamic accelerances respectively. First of all, the coupled microvibrations are obtained using the traditional method, where the WA static accelerances, $\mathbf{A}_{w}(\omega)$, are used in Eq. (5). Alternatively, the coupled microvibrations are obtained using the WA dynamic accelerances, $\mathbf{A}_{w}(\omega, \Omega)$. Both predicted coupled microvibrations are compared to the test results. RMS values of $\Phi_{C F x}, \Phi_{C F z F z}$ and $\Phi_{c M y M y}$ in $\boldsymbol{\Phi}_{F F_{-} \text {coupled }}$ are calculated at each speed. Results of $\Phi_{c F z F z}$ and $\Phi_{c M y M y}$ are shown in Fig. 14 as examples.

In Fig. 14(a), the predicted coupled microvibrations with WA static accelerances and WA dynamic accelerances are the same in the axial translational dof (i.e. the green line coincides with the red line). Also since nonlinearity is insignificant in axial translational dof, all results are matched very well in the complete speed band. In Fig. 14(b) on the other hand, the predicted coupled microvibrations in in-plane dofs have shown some noticeable differences compared to the test results. Even though, the predicted results with WA dynamic accelerances are closer to the test results. Note by including gyroscopic effects in WA accelerances, amplifications appear in the predicted results due to WA internal modes and hence larger RMS values at each speed compared to those predicted with WA static accelerances.

Also as an example, the predicted $\Phi_{c M y M y}$ at $2400 \mathrm{rev} / \mathrm{min}$ were extracted from the above figure and are compared to the corresponding test results. The microvibrations that were calculated using the standard microvibration analysis method (see Section 1 for the introduction) are plotted for comparison. They are together presented in Fig. 15.

Generally speaking, the coupled microvibrations predicted with WA static and dynamic accelerances have closely matched the test results in the considered frequency band. In particular, microvibrations predicted with WA dynamic accelerances are better matched to the coupled test results, especially at low frequencies (see the zoomed plot in Fig. 15). There are some significant differences in two regions in the figure: between $10 \mathrm{and} 20 \mathrm{~Hz}$ and between 40 and $60 \mathrm{~Hz}$ (the zoomed plot is not shown). They are identified as the places where gyroscopic effects become significant (see Fig. 13(c) for reference). The "rocking" mode mainly amplifies the hard-mounted microvibrations between $10 \mathrm{and} 20 \mathrm{~Hz}$ and thus the predicted coupled results. In the frequency region between 40 and $60 \mathrm{~Hz}$, amplifications are caused by the (beginning) interaction of the "rocking" mode and the in-plane translational mode, but its influences are not as obvious.

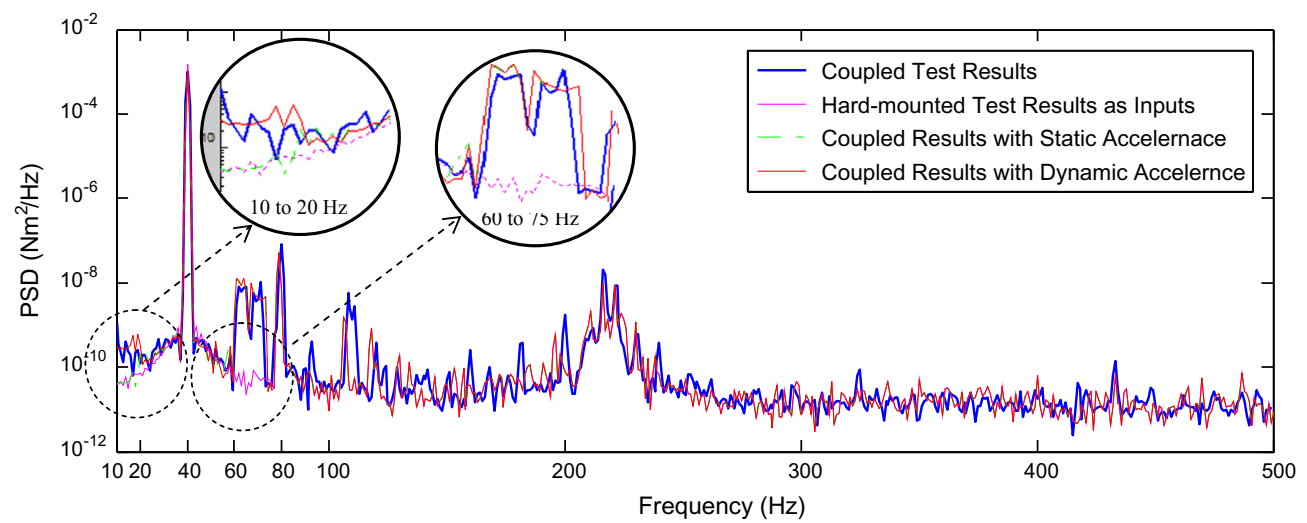

Fig. 15. Predicted coupled microvibrations and test results $\left(\Phi_{c M y M y}\right.$ at $\left.2400 \mathrm{rev} / \mathrm{min}\right)$. 
Comparing both predicted results to the hard-mounted microvibrations that are used directly as the inputs from the standard analysis method, not only are the amplifications below $20 \mathrm{~Hz}$ missed, but also are those between $60 \mathrm{and} 75 \mathrm{~Hz}$ owing to the lack of WA accelerances. At high frequencies however, the two predicted microvibrations with WA accelerances and hard-mounted microvibrations are the same as there are no WA modes in that region, and thus the inputs and outputs. Similar observations are also seen at other speeds, their results are plotted as the total RMS values in the speed band in Fig. 14.

From the results and discussions presented in this section, it is clear that the coupled microvibration analysis method with WA dynamic accelerances has advantage over the traditional method where WA static accelerance is used throughout the speed band. From a practicality point of view, since WA dynamic accelerances are obtained from the microvibration model, the complete analysis process can be programed following a systematic approach and thus can be modified for any type of WA as long as the validated microvibration model is available. However, it should be noted that due to nonlinearity within in-plane dofs at high spin speeds, the predicted coupled microvibrations are not reliable for the soft-suspended WA. Although nonlinearity has been included in the hard-mounted microvibrations as inputs, the analytical dynamic accelerance expressions of WA are impossible to model. Instead, for conventional designs (e.g. a rigid suspended WA typically spins between 0 and $5000 \mathrm{rev} / \mathrm{min}$ ) the analysis method introduced in this article is more beneficial.

\section{Conclusion}

This article presents the complete analysis of coupled microvibrations of a WA-structure system. In addition, this article describes a microvibration measurement system (SMVMS) to measure WA-induced microvibrations in coupled boundary condition that has also been developed to support this work. Various methods to reproduce the forces and moments generated by a cantilever configured WA when coupled with a supporting structure (seismic mass in this case) have been implemented and compared to the test results. The standard analysis method which simply uses hard-mounted microvibrations as the input loads to the supporting structures has been shown to neglect important features in the predicted responses. The traditional coupled analysis method which uses the accelerances of both WA (flywheel properties only) and supporting structure to correct the hard-mounted measurements, and also to reproduce the effects of the dynamic coupling, has been shown to give superior predictions to the test results. However, as this method does not include WA gyroscopic effects in the accelerance, in this article a procedure is proposed to calculate the accelerance including such effects. The results of the proposed method are compared to test results and show improvements over the other available analysis methods in the literature.

\section{Conflict of interest}

None.

\section{Acknowledgments}

The authors would like to thank SSBV Space \& Ground Systems UK and Surrey Satellite Technology Ltd (SSTL) for their support.

\section{Appendix A. Matrices of WA-seismic mass coupled system}

Below are the terms which appear in Eq. (3).

$$
\begin{aligned}
\mathbf{M}_{s} & =\left[\begin{array}{cccccccccc}
m_{w} & 0 & 0 & 0 & 0 & 0 & 0 & 0 & 0 & 0 \\
0 & m_{w} & 0 & 0 & 0 & 0 & 0 & 0 & 0 & 0 \\
0 & 0 & m_{w} & 0 & 0 & 0 & 0 & 0 & 0 & 0 \\
0 & 0 & 0 & I_{r} & 0 & 0 & 0 & 0 & 0 & 0 \\
0 & 0 & 0 & 0 & I_{r} & 0 & 0 & 0 & 0 & 0 \\
0 & 0 & 0 & 0 & 0 & m_{c} & 0 & 0 & 0 & 0 \\
0 & 0 & 0 & 0 & 0 & 0 & m_{c} & 0 & 0 & 0 \\
0 & 0 & 0 & 0 & 0 & 0 & 0 & m_{c} & 0 & 0 \\
0 & 0 & 0 & 0 & 0 & 0 & 0 & 0 & I_{c_{-} x x} & 0 \\
0 & 0 & 0 & 0 & 0 & 0 & 0 & 0 & 0 & I_{c_{-} y y}
\end{array}\right] \\
\mathbf{C}_{s} \approx & {\left[\begin{array}{cccccccccc}
c_{t} & 0 & 0 & 0 & -d c_{t} & -c_{t} & 0 & 0 & 0 & \\
0 & c_{t} & 0 & d c_{t} & 0 & 0 & -c_{t} & 0 & l c_{t} & 0 \\
0 & 0 & c_{z} & 0 & 0 & 0 & 0 & -c_{z} & 0 & 0 \\
0 & d c_{t} & 0 & d^{2} c_{t}+c_{r} & 0 & 0 & -d c_{t} & 0 & l d c_{t}-c_{r} & 0 \\
-d c_{t} & 0 & 0 & 0 & d^{2} c_{t}+c_{r} & d c_{t} & 0 & 0 & 0 & l d c_{t}-c_{r} \\
-c_{t} & 0 & 0 & 0 & d c_{t} & c_{t} & 0 & 0 & 0 & l c_{t} \\
0 & -c_{t} & 0 & -d c_{t} & 0 & 0 & c_{t} & 0 & -l c_{t} & 0 \\
0 & 0 & -c_{z} & 0 & 0 & 0 & 0 & c_{z} & 0 & 0 \\
0 & l c_{t} & 0 & l d c_{t}-c_{r} & 0 & 0 & -l c_{t} & 0 & l^{2} c_{t}+c_{r} & 0 \\
-l c_{t} & 0 & 0 & 0 & l d c_{t}-c_{r} & l c_{t} & 0 & 0 & 0 & l^{2} c_{t}+c_{r}
\end{array}\right] }
\end{aligned}
$$$$
\mathbf{G}_{s}=\left[\begin{array}{cccccccccc}
0 & 0 & 0 & 0 & 0 & 0 & 0 & 0 & 0 & 0 \\
0 & 0 & 0 & 0 & 0 & 0 & 0 & 0 & 0 & 0 \\
0 & 0 & 0 & 0 & 0 & 0 & 0 & 0 & 0 & 0 \\
0 & 0 & 0 & 0 & \Omega I_{z} & 0 & 0 & 0 & 0 & 0 \\
0 & 0 & 0 & -\Omega I_{z} & 0 & 0 & 0 & 0 & 0 & 0 \\
0 & 0 & 0 & 0 & 0 & 0 & 0 & 0 & 0 & 0 \\
0 & 0 & 0 & 0 & 0 & 0 & 0 & 0 & 0 & 0 \\
0 & 0 & 0 & 0 & 0 & 0 & 0 & 0 & 0 & 0 \\
0 & 0 & 0 & 0 & 0 & 0 & 0 & 0 & 0 & 0 \\
0 & 0 & 0 & 0 & 0 & 0 & 0 & 0 & 0 & 0
\end{array}\right]
$$$$
\mathbf{q}_{s}=\left\{\begin{array}{c}
x_{w} \\
y_{w} \\
z_{w} \\
\theta_{w} \\
\varphi_{w} \\
x_{c} \\
y_{c} \\
z_{c} \\
\theta_{c} \\
\varphi_{c}
\end{array}\right\}
$$ 


$$
\mathbf{K}_{s} \approx\left[\begin{array}{cccccccccc}
k_{t} & 0 & 0 & 0 & -d k_{t} & -k_{t} & 0 & 0 & 0 & -l k_{t} \\
0 & k_{t} & 0 & d k_{t} & 0 & 0 & -k_{t} & 0 & l k_{t} & 0 \\
0 & 0 & k_{z} & 0 & 0 & 0 & 0 & -k_{z} & 0 & 0 \\
0 & d k_{t} & 0 & d^{2} k_{t}+k_{r} & 0 & 0 & -d k_{t} & 0 & l d k_{t}-k_{r} & 0 \\
-d k_{t} & 0 & 0 & 0 & d^{2} k_{t}+k_{r} & d k_{t} & 0 & 0 & 0 & d l k_{t}-k_{r} \\
-k_{t} & 0 & 0 & 0 & d k_{t} & k_{t} & 0 & 0 & 0 & l k_{t} \\
0 & -k_{t} & 0 & -d k_{t} & 0 & 0 & k_{t} & 0 & -l k_{t} & 0 \\
0 & 0 & -k_{z} & 0 & 0 & 0 & 0 & k_{z} & 0 & 0 \\
0 & l k_{t} & 0 & l d k_{t}-k_{r} & 0 & 0 & -l k_{t} & 0 & l^{2} k_{t}+k_{r} & 0 \\
-l k_{t} & 0 & 0 & 0 & l d k_{t}-k_{r} & l k_{t} & 0 & 0 & 0 & l^{2} k_{t}+k_{r}
\end{array}\right]
$$$$
\mathbf{F}_{s}=\left\{\begin{array}{c}
-\sum_{i=1}^{n_{r t}} C_{i}^{r t} \Omega^{2} \sin \left(h_{i}^{r t} \Omega t\right) \\
\sum_{i=1}^{n_{r t}} C_{i}^{r t} \Omega^{2} \cos \left(h_{i}^{r t} \Omega t\right) \\
\sum_{i=1}^{n_{a t}} C_{i}^{a t} \Omega^{2} \sin \left(h_{i}^{a t} \Omega t\right) \\
\sum_{i=1}^{n_{r}} C_{i}^{r r} \Omega^{2} \cos \left(h_{i}^{r r} \Omega t\right) \\
\sum_{i=1}^{n_{r}} C_{i}^{r r} \Omega^{2} \sin \left(h_{i}^{r r} \Omega t\right) \\
0 \\
0 \\
0 \\
0 \\
0
\end{array}\right\}
$$

With reference to Fig. 2(b), the flywheel is modeled to be a rigid disk of mass $m_{w}$ and in-plane moment of inertia $I_{r}$, polar moment of inertia $I_{z}$. The flywheel is connected by a massless and rigid shaft of length $d$ to the soft-suspension system. The suspension system is modeled as a combination in five dofs. This includes two pairs of linear spring and dashpot, two pairs of torsional spring and dashpot (each in one of the two in-plane translational dofs $\left(x_{w}\right.$ and $\left.y_{w}\right)$ ) and a pair of linear spring and dashpot in the axial translational dof $\left(z_{w}\right)$. Due to axisymmetry of the WA, linear spring stiffness $k_{t}$ is the same in the two in-plane translational dofs and also for torsional spring stiffness $k_{r}$, dashpot damping coefficients $c_{t}$ and $c_{r}$. In axial translational dof, linear spring stiffness and dashpot damping coefficients are $k_{z}$ and $c_{z}$ respectively. In the forcing vector, microvibrations in each dof are modeled as a superposition of harmonics, where $C_{i}$ are amplitude coefficients of each harmonic, $h_{i}$ are harmonic numbers. Superscript "rt" indicates the in-plane translational dofs, "rr" the in-plane rotational dofs, and "at" the axial translational.

Note the matrices listed in Appendix A also represent those in Eq. (18) with subscript "ss" (flywheel-base system) replacing " $s$ " (WA-seismic mass system); properties of the WA base replace those of the seismic mass.

The physical damping ratios in radial translational dof, "rocking" dof and axial translational dof are extracted from the test results separately from each dof. Damping value expressions in $\mathbf{C}_{s}$ show that $c_{t}$ and $c_{z}$ are both independent from other dofs, therefore the physical damping ratios in these two dofs are directly related to each case. In "rocking" dof however, it is derived as $\varsigma_{R}=d^{2} s_{t}+\varsigma_{r}$. Since $d^{2} s_{t}$ is very small ( $d$ is $0.008 \mathrm{~m}$ and $s_{t}$ is 0.2$), \varsigma_{R} \approx \varsigma_{r}$, and therefore the physical damping ratio in "rocking" dof, $\varsigma_{R}$, is also directly related to $c_{r}$.

\section{Appendix B. Accelerances of flywheel-base coupled system}

$$
A_{w}=\left[\begin{array}{llllllllll}
A_{w 11} & A_{w 12} & 0 & A_{w 14} & A_{w 15} & A_{w 16} & A_{w 17} & 0 & A_{w 19} & A_{w 110} \\
A_{w 21} & A_{w 22} & 0 & A_{w 24} & A_{w 25} & A_{w 26} & A_{w 27} & 0 & A_{w 29} & A_{w 210} \\
0 & 0 & A_{w 33} & 0 & 0 & 0 & 0 & A_{w 38} & 0 & 0 \\
A_{w 41} & A_{w 42} & 0 & A_{w 44} & A_{w 45} & A_{w 46} & A_{w 47} & 0 & A_{w 49} & A_{w 410} \\
A_{w 51} & A_{w 52} & 0 & A_{w 54} & A_{w 55} & A_{w 56} & A_{w 57} & 0 & A_{w 59} & A_{w 510} \\
A_{w 61} & A_{w 62} & 0 & A_{w 64} & A_{w 65} & A_{w 66} & A_{w 67} & 0 & A_{w 69} & A_{w 610} \\
A_{w 71} & A_{w 72} & 0 & A_{w 74} & A_{w 75} & A_{w 76} & A_{w 77} & 0 & A_{w 79} & A_{w 710} \\
0 & 0 & A_{w 83} & 0 & 0 & 0 & 0 & A_{w 88} & 0 & 0 \\
A_{w 91} & A_{w 92} & 0 & A_{w 94} & A_{w 95} & A_{w 96} & A_{w 97} & 0 & A_{w 99} & A_{w 910} \\
A_{w 101} & A_{w 102} & 0 & A_{w 104} & A_{w 105} & A_{w 106} & A_{w 107} & 0 & A_{w 109} & A_{w 1010}
\end{array}\right] \text { WA Base DoFs }
$$

\section{References}

[1] European Space Agency, European Cooperation for Space Standardization (ESA ECSS), Chapter 13 Micro-gravity \& Micro-vibrations, Spacecraft Mechanical Loads Analysis, ECSS-E-HB-32-26, 2012.

[2] Z. Zhang, L. Yang, S. Pang, Jitter environment analysis for micro-precision spacecraft, Spacecraft Enviroment Engineering 26 (6) (2009) 528-534.

[3] M. Toyoshima, T. Jono, N. Takahashi, T. Yamawaki, K. Nakagawa, K. Arai, Transfer functions of microvibrational disturbances on a satellite, Proceedings of the 21st International Communications Satellite Systems Conference and Exhibit, Yokohama, Japan, 2003.

[4] O. Takahara, N. Yoshida, K. Minesugi, Microvibration transmissibility test of solar-B, Proceedings of the 24th International Symposium on Space Technology and Science, Miyazaki, Japan, 2004.

[5] S.E. Miller, P. Kirchman, J. Sudey, Reaction wheel operational impacts on the GOES-N jitter environment, AIAA Guidance, Navigation and Control Conference and Exhibit, South Carolina, USA, 2007.

[6] P. Laurens, E. Decoux, Microdynamic behaviour of momentum and reaction wheels, Proceedings of the Second Space Microdynamics and Accurate Symposium, Toulouse, France, 1997.

[7] R.A. Masterson, D.W. Miller, R.L. Grogan, Development of Empirical and Analytical Reaction Wheel Disturbance Models, Structures, Structural Dynamics and Materials Conference, St. Louis, MO, USA, 1999.

[8] R.A. Masterson, D.W. Miller, R.L. Grogan, Development and validation of reaction wheel disturbance models: empirical model, Journal of Sound and Vibration 249 (2002) 575-598.

[9] S.H. Oh, S.W. Rhee, Micro-vibration measurement, analysis and attenuation techniques of reaction wheel assembly in satellite, Journal of the Korean Society for Aeronautical \& Space Sciences 30 (8) (2002) 126-132. 
[10] S. Taniwaki, Y. Hatsutori, Y. Ohkami, Development of lower frequency disturbance detector for reaction wheel analysis, ASME Conference Proceedings, Vol. 2005, ASME, 2005, pp. 287-296.

[11] Y. Zhao, J. Sun, H. Tian, Development of methods identifying parameters in reaction wheel assembly disturbance model, Aircraft Engineering and Aerospace Technology 78 (4) (2006) 326-330.

[12] D.K. Kim, S.H. Oh, K.L. Yong, K.H. Yang, Numerical study on a reaction wheel and wheel-disturbance modeling, Journal of the Korean Society for Aeronautical \&' Space Sciences 38 (7) (2010) 702-708.

[13] H. Heimel, Spacewheel microvibration-sources, appearance, countermeasures, Proceedings of the Eighth International ESA Conference on Guidance E Navigation Control Systems, Karlovy Vary, Czech Republic, 2011.

[14] Z. Zhang, G.S. Aglietti, W. Zhou, Microvibrations induced by a cantilevered wheel assembly with a soft-suspension system, AIAA Journal 49 (5) (2011) 1067-1079.

[15] W. Zhou, G.S. Aglietti, Z. Zhang, Modelling and testing of a soft suspension design for a reaction/momentum wheel assembly, Journal of Sound and Vibration 330 (2011) 4596-4610.

[16] R.A. Masterson, D.W. Miller, Development and Validation of Empirical and Analytical Reaction Wheel Disturbance Models, MSc Thesis, Massachusetts Institute of Technology, 1999.

[17] L.M. Elias, D.W. Miller, A Structurally Coupled Disturbance Analysis Method using Dynamic Mass Measurement Techniques with Application to Spacecraft Reaction Wheel Systems, PhD Thesis, Massachusetts Institute of Technology, 2001.

[18] L.M. Elias, D.W. Miller, A coupled disturbance analysis method using dynamic mass measurement techniques, Proceedings of the 43rd AIAA/ASME/ASCE/ AHS/ASC Structures, Structural Dynamics, and Materials Conference, Denver, Colorado, 2002.

[19] Z. Zhang, W. Ren, G.S. Aglietti, Microvibration modeling, validation and coupled analysis of a reaction wheel in satellite, Proceedings of the European Conference on Spacecraft Structures, Materials \& Environmental Testing, Noordwijk, the Netherland, 2012.

[20] Y. Zhao, P. Zhang, W. Cheng, Measurement and study of disturbance characteristics of reaction wheel assembly, Journal of Experimental Mechanics 24 (6) (2009) 532-538.

[21] L.M. Elias, F.G. Dekens, I. Basdogan, L.A. Sievers, T. Neville, A Methodology for Modeling the Mechanical Interaction between a Reaction Wheel and a Flexible Structure, Interferometry in Space, Vol. 4852, SPIE, Waikoloa, HI, USA, 2003, pp. 541-555.

[22] I. Basdogan, L.M. Elias, F. Dekens, L. Sievers, Predicting the optical performance of the space interferometry mission using a modeling, testing, and validation, Journal of Vibration and Acoustics 129 (2) (2007) 148-157.

[23] L.M. Elias, D.W. Miller, Dynamics of Multi-body Space Interferometers including Reaction Wheel Gyroscopic Stiffening Effects: Structurally Connected and Electromagnetic Formation Flying Architectures, PhD Thesis, Massachusetts Institute of Technology, 2004.

[24] Z. Zhang, G.S. Aglietti, B.H. Le Page, Microvibration modelling and testing of a satellite reaction wheel assembly, Proceedings of the 10th International Conference on Vibrations in Rotating Machinery, London, UK, 2012. 